\title{
Constraints for the accretion disk evaporation rate in AGN from the existence of the Broad Line Region $\star$
}

\author{
B. Czerny ${ }^{1}$, A. Różańska ${ }^{1}$, and J. Kuraszkiewicz ${ }^{2}$ \\ ${ }^{1}$ Copernicus Astronomical Center, Bartycka 18, 00-716 Warsaw, Poland \\ e-mail: bcz@camk.edu.pl \\ 2 Harvard-Smithsonian Center for Astrophysics, 60 Garden Street, MS83, Cambridge, MA, USA
}

Received 21 March 2004 / Accepted 2 July 2004

\begin{abstract}
We analyze the consequences of the hypothesis that the formation of the Broad Line Region is intrinsically connected to the existence of the cold accretion disk. We assume that the Broad Line Region radius is reliably estimated by the formula of Kaspi et al. (2000, ApJ, 533, 631). We consider three models of the disappearance of the inner disk that limit the existence of the Broad Line Region: (i) the classical ADAF approach, i.e. the inner hot flow develops whenever it can exist; (ii) the disk evaporation model of Meyer \& Meyer-Hofmeister (2002, A\&A, 392, L5); (iii) the generalized disk evaporation model of Różańska \& Czerny (2000b, A\&A, 360, 1170). For each of the models, we determine the minimum value of the Eddington ratio and the maximum value of the broad line widths as functions of the viscosity parameter $\alpha$ and the magnetic field parameter $\beta$. We compare the predicted parameter space with observations of several AGN. Weak dependence of the maximum value of the $F W H M$ and minimum value of the Eddington ratio on the black hole mass in our sample is noticeable. It seems to favor the description of the cold disk/hot inner flow transition as in the classical ADAF approach rather than with the model of disk evaporation due to conduction between the disk and accreting corona.
\end{abstract}

Key words. accretion, accretion disks - galaxies: active - galaxies: Seyfert - X-rays: galaxies - quasars: emission lines

\section{Introduction}

One of the most important issues in studying accretion onto compact objects is to understand the process that causes some fraction of the accreting material to be heated sufficiently strongly to form a hot optically thin, predominantly thermal plasma in the vicinity of a black hole (see e.g. Narayan et al. 1998; Collin et al. 2001; Czerny 2003).

Observations of the X-ray emission from this plasma do not uniquely constrain the geometry of the plasma. Several phenomenological models are under consideration; those include magnetic flares above an accretion disk extending always down to the marginally stable orbit (Galeev et al. 1979; Haardt et al. 1994), or the solutions of accretion disk structure with inner optically thin (possible advection-dominated) flow (Ichimaru 1977; Narayan \& Yi 1994; Abramowicz et al. 1995). The geometry possibly depends on the luminosity to the Eddington luminosity ratio in a given object. Detailed XMM-Newton and Chandra studies of the X-ray reflection (both continuum and iron $\mathrm{K}_{\alpha}$ line) suggest that at high Eddington ratios the disk extends to the marginally stable orbit and its surface appears strongly ionized (e.g. Różańska et al. 2004 for Ton S180; Janiuk et al. 2001 and Pounds et al. 2003 for PG 1211+143;

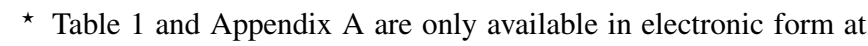
http://www . edpsciences.org
Sako et al. 2003 for MCG -6-30-15 and Markarian 766) while in low Eddington ratio sources the spectra are well-described as being due to cold reflection from the outer part of the accretion disk (e.g. O'Brien et al. 2003).

Those observations motivate the development of models in which the material slowly evaporates from the cold disk into a hot corona as the black hole is approached (Liu et al. 1999, 2002; Różańska \& Czerny 2000b; Meyer \& Meyer-Hofmeister 2002; Meyer-Hofmeister \& Meyer 2003). All such models predict that at high Eddington ratios the evaporation effect is relatively inefficient so the cold optically thick disk survives all the way down to the black hole while for low Eddington ratio sources the evaporation leads to a complete evacuation of the disk and formation of an inner optically thin, hot flow.

However, the physical description of the evaporation rate involves a number of complicated processes, and various models rely on several underlying assumptions so it is very important to verify the models against observational constraints. In this paper we use the constraint based on the measured width of the broad emission lines. The exact nature of the broad line region (BLR) is not known but most models connect the formation of low ionization lines like $\mathrm{H}_{\beta}$ with the wind developing close to the disk surface (Collin-Souffrin et al. 1988; Dultzin-Hacyan et al. 2000; Laor 2003a). If such an approach is adopted, the existence of the broad lines at a certain range of 
radii is possible only if the cold disk extends also at least to the same radial range, and regions of the efficient line/wind formation are related to specific phenomena taking place within the disk. For example, such an approach led Elvis (2000) to propose a general, consistent scheme for quasar structure.

The details of the processes, including disk/BLR coupling, are by no means clear but the large amount of the existing data opens possibilities to test various hypotheses of the nature of this relation. Laor (2003b) noticed that there appears to be an upper limit to the broad line width of about $25000 \mathrm{~km} \mathrm{~s}^{-1}$ which apparently sets a lower limit for the luminosity of the broad line object. He postulated that a significant population of AGN exists that intrinsically do not have broad lines. Further developing this idea, Nicastro et al. (2003) found the division between the AGN with and without broad lines occurs at the Eddington ratio of $\sim 0.001$, and interpreted this result as an argument for a connection between the onset of a wind from the disk surface and the transition from a gas dominated to a radiation pressure dominated part of the cold disk.

In this paper we assume that the outer cold disk turns into an inner hot, optically thin and radiatively inefficient flow. We further assume that the BLR may form above a cold disk, in its atmosphere or outflowing wind, but not above a hot inner flow. These assumptions allow us to qualitatively test the theoretical predictions for the location of such a transition. We consider three specific models: the classical condition for the existence of ADAF (Abramowicz et al. 1995), the evaporation model of Meyer \& Meyer-Hofmeister (2002) and the evaporation model of Różańska \& Czerny (2000) generalized for the case of a nonnegligible magnetic field.

\section{Method}

\subsection{Size of the BLR}

The size of the broad line region depends predominantly on the luminosity of the active nucleus. The most accurate results are based on the reverberation method. Delays of the $\mathrm{H}_{\beta}$ response with respect to the changes of the continuum lead to the relation (Kaspi et al. 2000)

$R_{\mathrm{BLR}}=32.9\left(\frac{\lambda L_{\lambda}(5100 \AA)}{10^{44}}\right)^{0.7} 1 \mathrm{lt}$ days,

with a relatively high correlation coefficient in the luminosity range covering over three decades (with most sources between $10^{42}$ and $10^{45} \mathrm{erg} \mathrm{s}^{-1}$ ).

The value of the monochromatic luminosity at $5100 \AA$ is surprisingly closely related to the bolometric luminosity of an AGN

$L_{\mathrm{bol}}=9 \lambda L_{\lambda}(5100 \AA)$.

(e.g. Kaspi et al. 2000; Collin et al. 2002).

The bolometric luminosity can be measured in dimensionless Eddington units, where

$L_{\mathrm{Edd}}=\frac{4 \pi G M m_{\mathrm{p}} c}{\sigma_{\mathrm{T}}}$.
We express the mass in units of $10^{8} M_{\odot}$ (i.e. $M_{8}=M / 10^{8} M_{\odot}$ ). Finally, we give the BLR radius in the units of Schwarzschild radius, $R_{\text {Schw }}=2 G M / c^{2}$, since theoretical estimates usually refer to such a quantity:

$R_{\mathrm{BLR}}=1.8 \times 10^{4}\left(\frac{L_{\mathrm{bol}}}{L_{\mathrm{Edd}}}\right)^{0.7} M_{8}^{-0.3} R_{\mathrm{Schw}}$.

\subsection{Line width}

The determination of the black hole mass by Kaspi et al. (2000) was based on the assumption that the motion of the BLR clouds is predominantly Keplerian. This approach was supported by a separate analysis of the several emission lines in NGC 5548 by Peterson \& Wandel (1999). To determine the FWHM from the Keplerian velocity the correction factor $2 / \sqrt{3}$ is needed, which accounts for velocities in three dimensions and for the full width being two times higher than the velocity dispersion, i.e.:

$F W H M=\frac{2}{\sqrt{3}} \sqrt{\frac{G M}{R_{\mathrm{BLR}}}}$.

Here, we reverse the approach of Kaspi et al. (2000), and we express the width of the $\mathrm{H}_{\beta}$ line as a function of the black hole mass and its luminosity

$F W H M=1824 M_{8}^{0.15}\left(\frac{L_{\mathrm{bol}}}{L_{\mathrm{Edd}}}\right)^{-0.35}\left[\mathrm{~km} \mathrm{~s}^{-1}\right]$.

\subsection{Evaporation radius}

The location of the transition from the optically thick disk embedded in a hot corona into a single phase inner ADAF depends on the assumptions underlying the evaporation process. The transition radius, in Schwarzschild units, depends on the dimensionless accretion rate defined as

$\dot{m}=\frac{\dot{M}}{\dot{M}_{\mathrm{Edd}}} ; \quad \dot{M}_{\mathrm{Edd}}=\frac{4 \pi G M m_{\mathrm{p}}}{\sigma_{\mathrm{T}} c \eta_{0}}$,

where $\eta_{0}=1 / 12$ is the efficiency of disk accretion in the Newtonian approximation.

In classical papers about the advection dominated flows (ADAFs), where the process of disk evaporation was not considered, the estimate of the truncation radius was simply based on the absence of a strong ADAF solution above this radius (Abramowicz et al. 1995; Honma 1996; Kato \& Nakamura 1998):

$R_{\text {evap }}^{A}=2.0 \dot{m}^{-2} \alpha_{0.1}^{4} R_{\mathrm{Schw}}$.

The unique relation between truncation radius and mass accretion rate can be determined when evaporation of the cold disc is taken into account. In the model of a single-temperature accreting corona with the evaporation efficiency determined by the conduction between the cold disk and a hot corona described as is customarily done for the solar corona, this transition takes place at the evaporation radius:

$R_{\text {evap }}^{*}=18.3 \dot{m}^{-0.85} R_{\text {Schw }}$ 
(Liu et al. 1999). This relation is derived for a single value of the viscosity parameter $\alpha=0.3$.

The same model, but generalized for the presence of magnetic field within the accreting corona can be roughly parameterized as

$R_{\text {evap }}^{B}=18.3 \dot{m}^{-0.85} \beta^{2.5} R_{\text {Schw }}$,

which is based on the numerical results presented by Meyer $\&$ Meyer-Hofmeister (2002). Here $\beta$ is the ratio of the gas pressure to the total (gas + magnetic) pressure in the corona. Therefore, the ratio of gas to the magnetic pressure used in Meyer \& Meyer-Hofmeister (2002) is $P_{\mathrm{g}} / P_{\mathrm{m}}=\beta /(1-\beta)$.

In the two-temperature model of accreting corona with the evaporation efficiency calculated under a constant pressure assumption with conduction self-consistently determined from radiative processes (Różańska \& Czerny 2000a), this transition is predicted to be at

$R_{\text {evap }}^{* *}=19.5 \dot{m}^{-0.53} \alpha_{0.1}^{0.8} R_{\text {Schw }}$.

This relation shows a different dependence on the viscosity parameter than that predicted in Różańska \& Czerny (2000b), due to an error in the original paper.

We also generalize this model for the case of the contribution of the magnetic pressure to the total pressure (see Appendix A). We repeat the analysis of Różańska \& Czerny (2000b). Numerical results for the case of low accretion rate (i.e. negligible condensation) can be approximated analytically as

$\dot{m}_{z}=\dot{m}_{z}^{0} \beta$

and consequently the evaporation radius changes to

$R_{\text {evap }}^{C}=19.5 \dot{m}^{-0.53} \alpha_{0.1}^{0.8} \beta^{-1.08} R_{\text {Schw }}$.

\subsection{Accretion efficiency}

The bolometric luminosity is in turn related to the accretion rate through the accretion efficiency

$L_{\mathrm{bol}}=\eta \dot{M} c^{2}$.

In Eq. (7) we used the efficiency $\eta_{0}$ which is appropriate for the description of outer parts of the flow well represented by a classical disk accretion. However, the actual efficiency of the conversion of the mass accretion into observed luminosity in the innermost part of the flow may be much lower due to advection. Therefore, we consider the efficiency $\eta$ in a general form

$\eta=\eta_{0} \mathcal{F}$,

and we later specify $\mathcal{F}$ as a function of model parameters. In such a general case the bolometric luminosity depends on the dimensionless accretion rate as:

$\frac{L_{\text {bol }}}{L_{\mathrm{Edd}}}=\dot{m} \mathcal{F}$.

The existence of the evaporation radius affects the efficiency of the accretion. Without an inner advection-dominated flow the

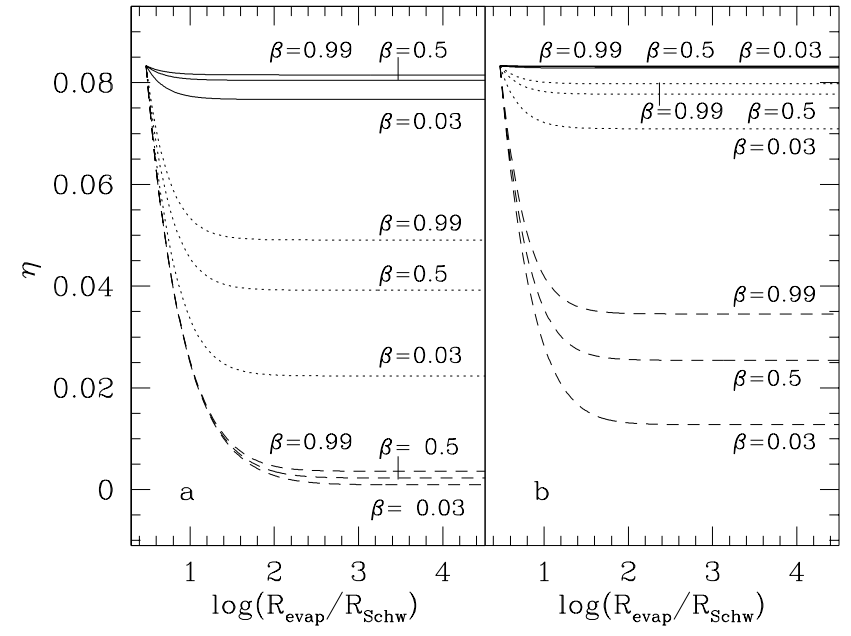

Fig. 1. The dependence of accretion efficiency on evaporation radius according to Eq. (18) for two different viscosity parameters $\alpha=0.1$ (panel a)), and $\alpha=0.04$ (panel b)). Solid lines represent the case of $\dot{m}=0.3$, dotted lines $-\dot{m}=0.03$, and the dashed lines $-\dot{m}=0.003$.

correction factor to the efficiency $\mathcal{F}$ in Eqs. (4) and (6) is equal to 1 , and the evaporation radius is equal to 3 , in Schwarzschild units, for a non-rotating black hole. This efficiency drops with an increasing $R_{\text {evap }}$ since the number of soft photons penetrating the hot region decreases with an increase of its radial optical depth. For large values of $R_{\text {evap }}$ this efficiency finally saturates since there, the locally emitted synchrotron and bremsstrahlung photons are the only source of soft photons for Comptonization. According to the Monte Carlo computations of Kurpiewski \& Jaroszyński (2000) this saturation value is on the order of $\varepsilon$, where:

$\varepsilon=170.99\left(\frac{\dot{m}}{\alpha_{0.1}^{2}}\right)^{1.5}(1.25 \beta+0.375)$.

In order to approximate this complex behaviour we use a simple formula for the correction factor mentioned above, having in mind that $r_{\text {evap }}=R_{\text {evap }} / R_{\text {Schw }}$

$\mathcal{F}=\frac{3}{r_{\text {evap }}}+\left(1-\frac{3}{r_{\text {evap }}}\right) \frac{\varepsilon r_{\text {evap }}}{3+\varepsilon r_{\text {evap }}}\left(\frac{\varepsilon}{\varepsilon+1}\right)$,

which gives the correct asymptotic dependence for both small and large evaporation radii.

The dependence of the total accretion efficiency described by Eqs. (15) and (18) on the evaporation radius is shown in Fig. 1. As requested, this efficiency reduces to $1 / 12$ if no evaporation takes place $\left(r_{\text {evap }}=3\right.$ ), it drops for increasing $r_{\text {evap }}$ due to the decreasing contribution of the cold disk emission and finally it saturates at the value characteristic of the ADAF solution itself. This last value depends significantly both on the viscosity parameter (directly responsible for angular momentum transfer) and on the magnetic field strength parameter $\beta$ (regulating the electron cooling through the synchrotron emission). 


\subsection{Minimum Eddington ratio and maximum line width for broad line objects}

We assume that broad emission lines cannot form in the radial range inside the evaporation radius. Therefore the equality

$R_{\text {evap }}=R_{\mathrm{BLR}}$

gives the minimum Eddington ratio for which the BLR can exist for a given black hole mass $M$, assuming a certain value of the viscosity parameter $\alpha$ and/or of the magnetic pressure contribution $\beta$.

The same condition, combined with Eq. (6) gives the maximum value of the $F W H M$ of $\mathrm{H}_{\beta}$, again for a given $M, \alpha$ and/or $\beta$.

Both $F W H M$ of $\mathrm{H}_{\beta}$ and the Eddington ratio can be obtained or estimated on the basis of the observational data. When complemented with black hole mass estimates for each source, the measured values provide constraints for $\alpha$ and/or $\beta$.

\section{Results}

\subsection{BLR radii and disk evaporation radii in three models}

The plots of $R_{\mathrm{BLR}}$ and $R_{\text {evap }}$ as functions of the Eddington ratio for the considered models are shown in Fig. 2. The position of the straight line representing $R_{\mathrm{BLR}}$ is the same for all models and depends only on the mass of the object (see Eq. (4)). The evaporation radius depends on the model.

In the upper panel of Fig. 2 we show the results based on the strong ADAF principle (model A) for three values of the viscosity parameter $\alpha$ and two extreme values of the parameter $\beta$. Lowering the value of the viscosity strongly decreases the inner ADAF region (see Eq. (8)). The weak dependence on $\beta$ is caused by the change in accretion flow efficiency in the ADAF part (see Eq. (17)).

The intersection of $R_{\mathrm{tr}}^{A}$ and $R_{\mathrm{BLR}}$ determines the minimum Eddington ratio, for a given $\alpha$ and $\beta$, for which the BLR exists: below this value the BLR would form in the ADAF zone. Therefore, again for a given $\alpha$, the size of the BLR as a function of accretion rate is defined as a fragment of the $R_{\mathrm{BLR}}$ line above the crossing point. For a $10^{8} M_{\odot}$ object and $\alpha \sim 0.03$, this minimum Eddington ratio of a broad line object is about 0.005 , and the minimum value of $R_{\mathrm{BLR}}$ is about $400 R_{\mathrm{Schw}}$, corresponding to the maximum value of the $F W H M$ equal to $12200 \mathrm{~km} \mathrm{~s}^{-1}$. The minimum Eddington ratio increases and the FWHM maximum decreases with increasing $\alpha$.

In the middle panel of Fig. 2 we plot the results for Model $\mathrm{B}$, varying the parameter $\beta$. In this case the effect of $\beta$ is much stronger than in the ADAF approach since the magnetic field affects the evaporation efficiency directly (see Eq. (10)), apart from the change of hot flow efficiency. A weaker magnetic field (higher $\beta$ ) allows the disk to extend closer in. The minimum Eddington rate of a broad line object is predicted to be $\sim 0.003$ for low magnetic field and $\sim 10^{-4}$ for $\beta=0.1$. The lack of dependence on $\alpha$ is caused by the arbitrary assumption of $\alpha=0.3$ made by Meyer \& Meyer-Hofmeister (2002), with no results available for other values of the viscosity parameter.
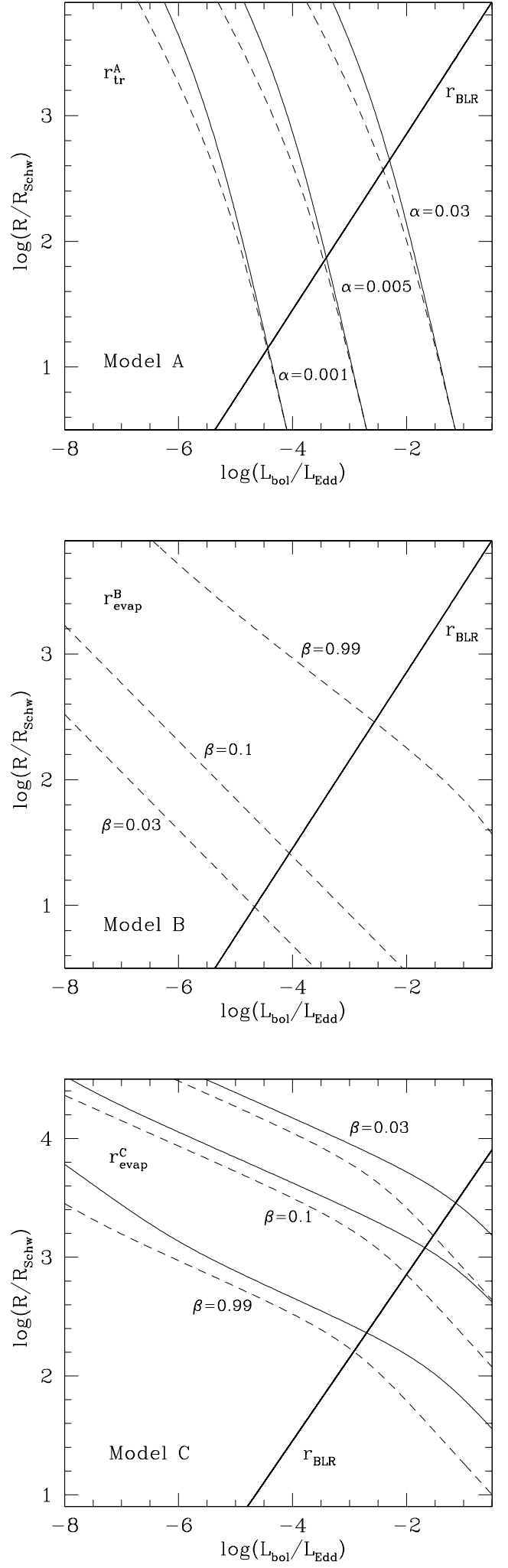

Fig. 2. The dependence of the BLR radius (thick continuous line) and the transition radius (thin lines) on the external accretion rate in model A (upper panel), model B (middle panel) and model C (lower panel) for the black hole mass $10^{8} M_{\odot}$. Model A parameters: $\beta=0.99$ (continuous thin lines), and $\beta=0.03$ (dashed thin lines). Model B parameters: $\alpha=0.3$ (dashed lines). Model C parameters: $\alpha=0.1$ (continuous thin lines), and $\alpha=0.02$ (dashed thin lines). The intersection determines the minimum Eddington ratio for a broad line object and the minimum BLR radius. 


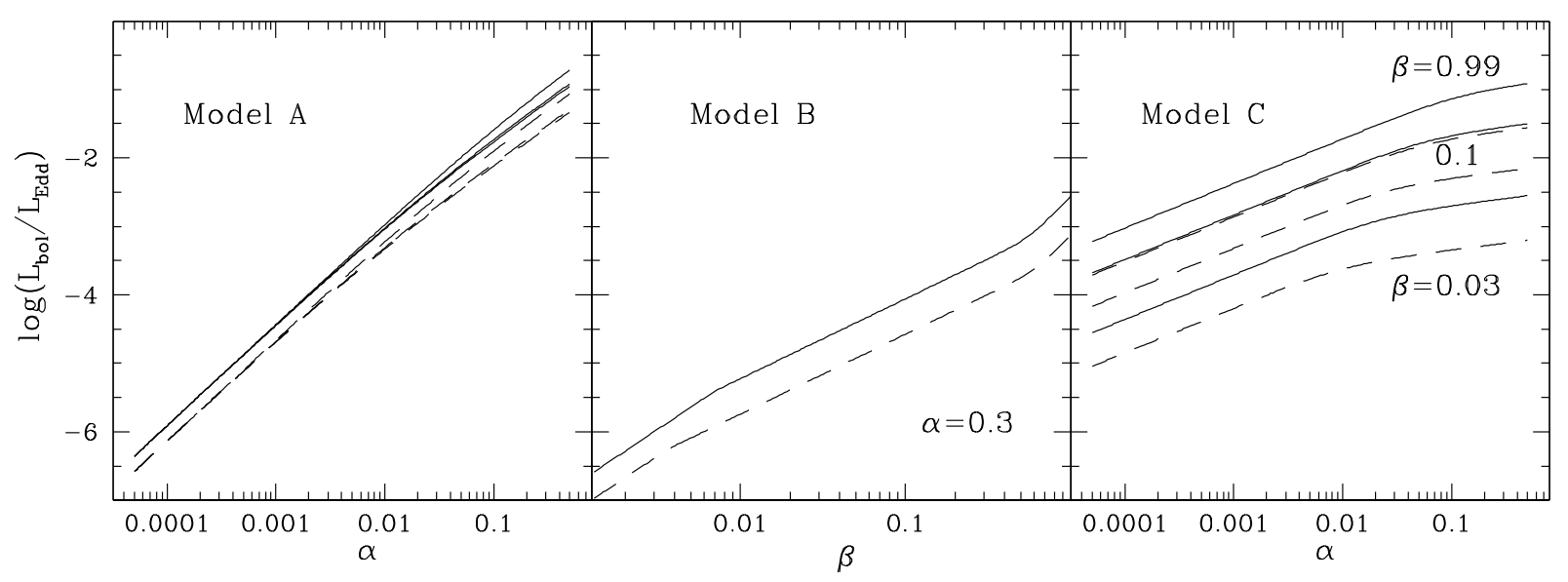

Fig. 3. The dependence of the minimum of the Eddington rate for three models A (left), B (middle), and C (right panel) on viscosity and magnetic parameters. Note that model B is computed only for $\alpha=0.3$ (see text). Solid lines represent the case of $M_{\mathrm{BH}}=10^{8} M_{\odot}$, while dashed lines $-10^{6} M_{\odot}$.

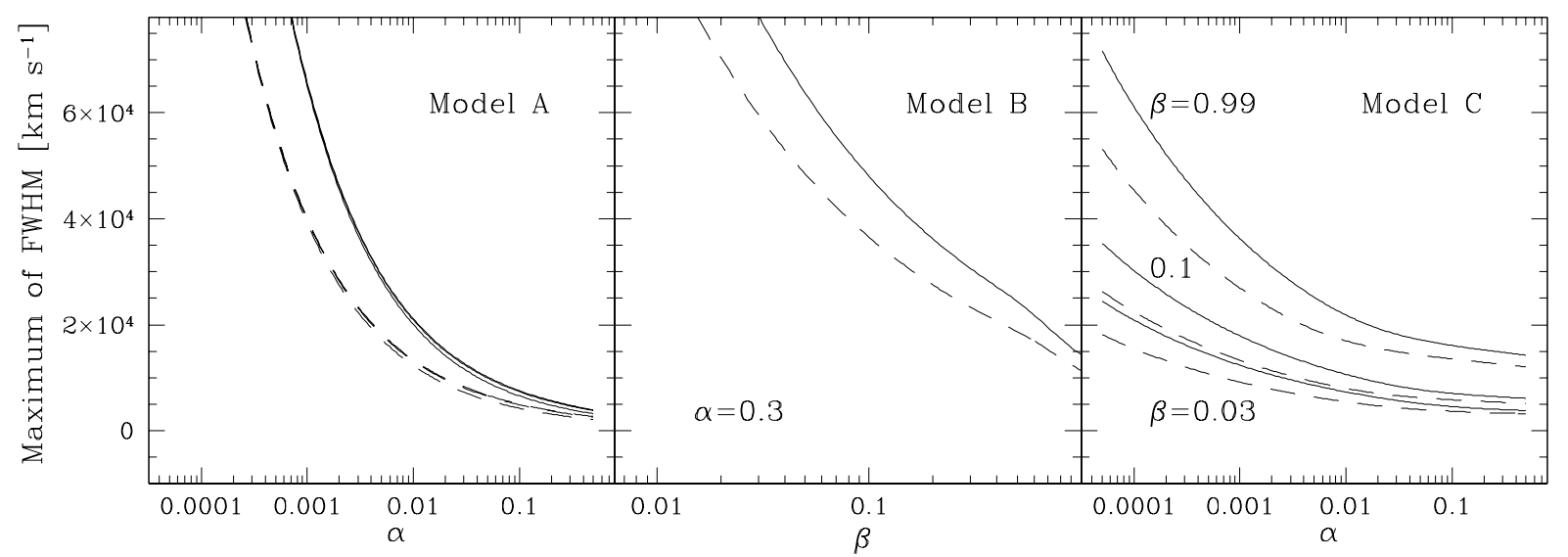

Fig. 4. The dependence of the maximum of FWHM for three models A (left), B (middle), and C (right panel) on viscosity and magnetic parameters. Note that model B is computed only for $\alpha=0.3$ (see text). Solid lines represent the case of $M_{\mathrm{BH}}=10^{8} M_{\odot}$, while dashed lines $10^{6} M_{\odot}$.

The results for the model $\mathrm{C}$ are shown in the lower panel of Fig. 2. Here we have a strong dependence on both $\alpha$ and $\beta$ since again the magnetic field plays an active role in the process of the disk evaporation (see Eq. (13) and the Appendix). Generally, larger magnetic field leads to more efficient disk evaporation and consequently the formation of the BLR closer to the black hole is strongly limited.

\subsection{Minimum Eddington rate of a broad line object}

The minimum Eddington rate of a broad line object is defined by the line crossings in Fig. 2. It depends on the black hole mass, viscosity and the magnetic field. We summarize some of these relations in Fig. 3.

The values obtained from the classical ADAF solution are very low unless the viscosity parameter is of the order of 0.01 or larger, and the dependence on the magnetic field is negligible (see Fig. 3, left panel). Derived limits formally depend on the assumed black hole mass (Fig. 2 was constructed for $M_{\mathrm{BH}}=10^{8} M_{\odot}$, but the analysis can be repeated for other black hole masses). However, in the case of the ADAF model, this dependence is rather weak.

The values obtained from model B also span the whole range, increasing significantly with an increase of the magnetic field. Larger black hole mass results in a larger value of the minimum bolometric luminosity of a BLR object.

In the case of model $\mathrm{C}$ the dependence on all parameters, $\alpha, \beta$ and the black hole mass is important. The Eddington ratio plot is generally flatter, and even for very low viscosity and a strong magnetic field the minimum Eddington ratio is larger than $\sim 10^{-5}$.

\subsection{Maximum line width of a broad line object}

The size of the BLR corresponds to the $F W H M$ of the $\mathrm{H}_{\beta}$ line (see Eq. (5)), so we can replace the radius with the values of $F W H M$ which is directly measured from observations. Therefore, the allowed region in the $R-L / L_{\text {Edd }}$ diagram translates into the equivalent region in the $F W H M-L / L_{\text {Edd }}$ diagram according to Eq. (6). Alternatively, to represent these limits we 
can plot the maximum $F W H M$ as a function of the adopted parameters.

The resulting maximum of the $F W H M$ as a function of $\alpha$ or $\beta$ is shown in Fig. 4. All models show clear dependence of the limits on the black hole mass (dashed and continuous lines for $10^{6} M_{\odot}$ and $10^{8} M_{\odot}$, correspondingly)

Classical ADAF solution allows for very broad lines if the viscosity is small. Model B also displays large parameter range coming from adoption of weak or strong magnetic field. In the case of model $\mathrm{C}$ the dependence of the $F W H M$ is shallower and very broad lines are expected only for very low viscosity and very weak magnetic field; otherwise, evaporation is too efficient for a cold disk to survive close to a black hole.

\section{Comparison with observations}

Theoretical constraints for the properties of the broad line objects can be compared with the observed properties. We attempt to perform such a comparison using the sample of objects collected from the literature. The measured quantities as well as estimated black hole masses and Eddington rates for these objects are summarized in Table 1. These objects, taken from various samples, do not form an unbiased sample of AGN so the conclusions cannot be firm. Nevertheless, such an analysis shows the effectiveness of the method and offers some indicative results.

\subsection{Data}

Optical/UV spectra of LBQS (large Bright Quasar Survey) objects included in the sample of Forster et al. (2001) were studied with MMT and du Point telescopes. We selected objects for which reliable MgII line measurements were performed, supplemented by the measurement of the continuum at $2800 \AA$, rest frame. The measured flux was corrected for the Galactic reddening.

The mass of the central black hole was calculated from the relation found by Kaspi et al. (2000)

$M=4.817\left(\frac{\lambda L_{\lambda}(5100 \AA)}{10^{44}}\right)^{0.7} F W H M^{2} M_{\odot}$.

We determined the $\lambda L_{\lambda}(5100 \AA)$ from the measured flux at $2800 \AA$ by extrapolating the measured flux to $5100 \AA$, assuming a power law spectrum with a slope 0.5 (i.e. $F_{v} \propto v^{-0.5}$ ). The cosmological model used throughout the paper is $H_{0}=$ $75 \mathrm{~km} \mathrm{~s}^{-1} \mathrm{Mpc}^{-1}$ and $q_{0}=0.5$. We used Eq. (2) to determine the bolometric luminosity of an object, and subsequently its Eddington ratio, $L_{\text {bol }} / L_{\text {Edd }}$, with $L_{\text {Edd }}$ defined by Eq. (3). The measured quantities ( $F W H M$ and $\left.F_{\lambda}\right)$, the derived black hole mass and the Eddington ratio are given in Table 1.

Similarly, for objects from the sample of Kuraszkiewicz et al. (2002) and Kuraszkiewicz et al. (2004) the FWHM of the MgII line was measured together with the continuum at $2800 \AA$, rest frame. The measurements were performed for HST data. The black hole mass of these sources and the Eddington ratio were derived as above.
For objects taken from Kaspi et al. (2000), as well as for those originally included in the study of Wandel et al. (1999), the measurements of $F W H M$ of $\mathrm{H} \beta$ lines are available, with the exception of PG $1351+640$ for which an $\mathrm{H}_{\alpha}$ measurement was given, but corrected by $20 \%$ to account for a systematic difference between $\mathrm{H}_{\alpha}$ and $\mathrm{H}_{\beta}$ line widths. The luminosity of the objects was provided in the form of $\lambda L_{\lambda}$ at $5100 \AA$, assuming the Hubble constant $H_{0}=75 \mathrm{~km} \mathrm{~s}^{-1} \mathrm{Mpc}^{-1}$ and the deceleration parameter $q_{0}=0.5$. The values of the black hole masses for these objects were derived from the reverberation measurements and were given by Kaspi et al. (2000), and we determine the Eddington ratio using the bolometric luminosity estimate of Eq. (2).

For the nearby quasars taken from Shang et al. (2003) the $F W H M$ of $\mathrm{H} \beta$ was given together with $L_{v}(1549 \AA)$. This last value was given for $H_{0}=50 \mathrm{~km} \mathrm{~s}^{-1} \mathrm{Mpc}^{-1}$ and $q_{0}=0.5$ so it was necessary to recalculate it to $H_{0}=75 \mathrm{~km} \mathrm{~s}^{-1} \mathrm{Mpc}^{-1}$ adopted in our paper. The black hole mass and the Eddington ratio were obtained as for Forster et al. and Kuraszkiewicz et al. objects.

We also include several more distant quasars from Brotherton et al. (1994a). We include in our sample only the radio-quiet sources. The MgII widths were measured for those sources, and the continuum in magnitudes measured at $2200 \AA$ was provided by Steidel \& Sargent (1991). The magnitude values were reversed into the flux measurement using the definition of Steidel \& Sargent (1991), and subsequently the absolute flux at $2200 \AA$ was derived assuming the same cosmological model as before. The black hole mass and the Eddington ratio were determined as for Forster et al. and Kuraszkiewicz et al. objects.

\subsection{Results}

The Eddington ratios of practically all objects from Table 1 are larger than 0.01. All these objects are classified as typical Broad Line objects: QSO, Seyfert 1 galaxies or Narrow Line Seyfert 1 galaxies. The maximum values of the FWHM among the objects in our sample do not exceed $17000 \mathrm{~km} \mathrm{~s}^{-1}$, well within the conservative upper limit for a Broad Line object adopted by Laor (2003b) in his discussion. Therefore, our sample supports the conclusion that Broad Line objects exist above a certain threshold in the Eddington ratio, and the widths of their broad lines are limited. Such an interpretation is consistent with an accretion scenario based on the evaporation of the inner cold disk in objects with a low Eddington ratio.

In order to determine which of the three specific models (see Sect. 2.3) better represents the allowed parameter space for the Broad Line objects we use two diagrams. In Fig. 5 we show the dependence between the line width and the Eddington ratio for objects from our sample. Objects coming from various subsamples are marked with different symbols but they all form a mostly uniform distribution. The black dot marking an object with a rather large $F W H M\left(15000 \mathrm{~km} \mathrm{~s}^{-1}\right)$ and low Eddington ratio (0.0022) represents the source Arp 102B (the second to last source from Reference 3 in Table 1), which belongs to the 


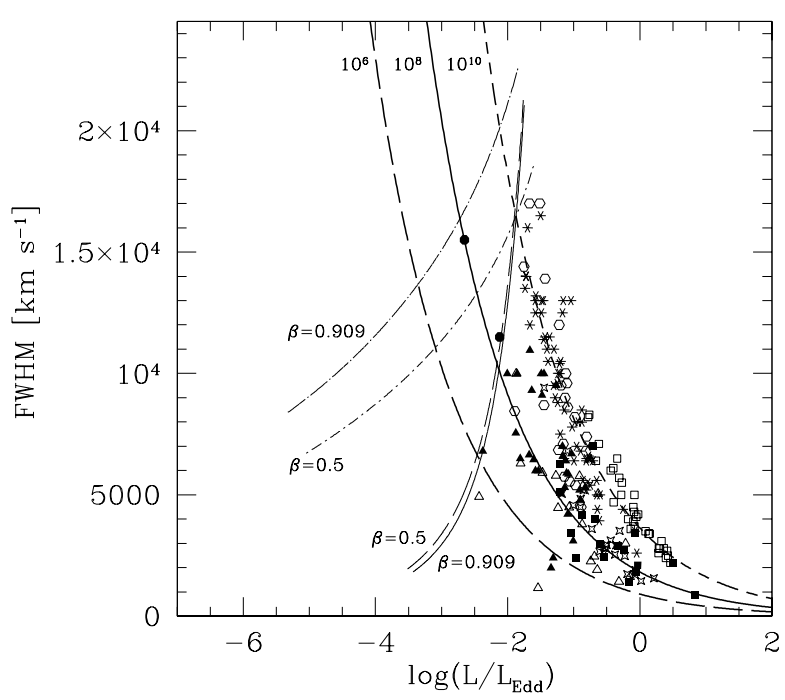

Fig. 5. The dependence of full width of half maximum of the broad line on bolometric luminosity. Observational points come from Table 1. Lines marked with the corresponding values of the black hole mass show the trends given by Eq. (6). Other lines constrain the parameter space of the Broad Line objects: continuous line and longdash line represent the ADAF (model A) with the viscosity $\alpha=0.04$ in the case of negligible magnetic field and for $\beta=0.5$, correspondingly; dashed lines show the limits based on model $\mathrm{C}$ assuming viscosity parameter $\alpha=0.02$ and again two values of $\beta$ ( 0.909 and 0.5 , correspondingly).

class of radio loud objects with double peaked broad emission lines (Chen \& Halpern 1989).

We did not plot error bars in Fig. 5 since they are rather difficult to asses. The measurements of the FWHM are performed with 20-30 percent accuracy; the Eddington ratio is in most cases derived on the basis of statistical correlations like those given by Eqs. (2) and (20) which show a certain intrinsic scatter.

The objects basically follow the relation between the line width and the Eddington ratio given by Eq. (6) marked in Fig. 5 for three specific values of the black hole mass. Therefore, the relation found by Kaspi et al. (2000) seems to describe the whole sample of objects quite well. The upper limits for the line width at a given Eddington ratio, estimated from two models, are marked as lines roughly perpendicular to the Kaspi et al. relations. A successful model should clearly separate the region occupied by objects from the avoidance region.

We plot four limiting lines coming from two models. The ADAF solution (model A) is represented by two lines: a continuous line for a negligible magnetic field and long dashed line for $\beta=0.5 ; \alpha=0.04$ in both cases. Evaporation model $\mathrm{C}$ is represented by two other lines: a long-dash-dot line (negligible magnetic field) and a short-dash-dot line $(\beta=0.5) ; \alpha=0.02$ in both cases.

Here it is apparent that all theoretical constraints for the line width are not universal, but show some dependence on the Eddington ratio: the line is allowed to be broader for an object with a lower mass/lower Eddington ratio. However, the strength of this coupling depends on the model. In the ADAF model the limiting line is quite steep and the upper limit from the line

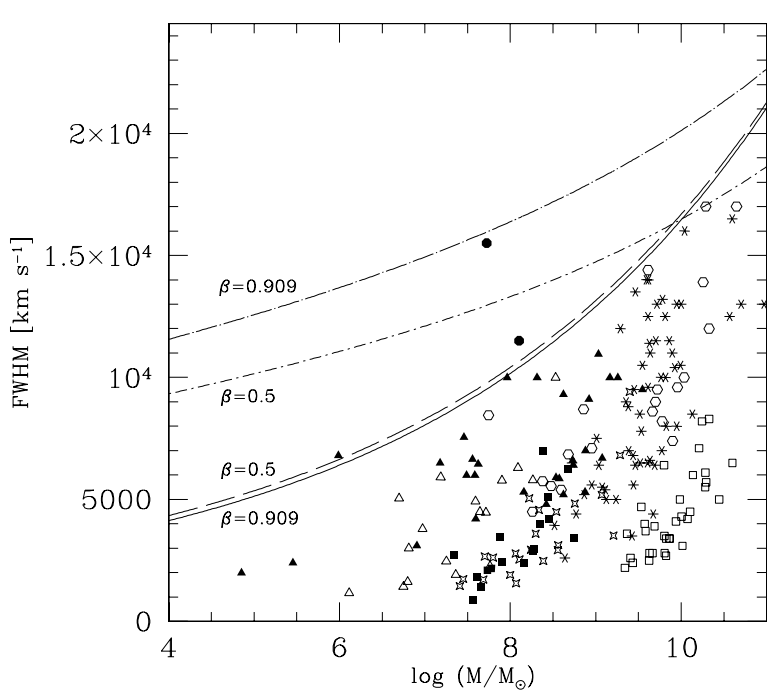

Fig. 6. The dependence of full width of half maximum on the black hole mass. Observational points come from Table 1. Continuous and long-dashed lines represent the limits based on model A, viscosity parameter $\alpha=0.04, \beta=0.909$ and $\beta=0.5$, correspondingly. Shortdash-dot line and long-dash-dot lines are for $\beta=0.5$ and $\beta=0.909$.

width is almost universal while in the evaporation model $\mathrm{C}$ the line is flatter and the predicted limiting value for the line width depends considerably on the object mass and Eddington ratio. Meyer-Hofmeister \& Meyer (2001) have shown some calculations of evaporation rate for different values of the viscosity parameter. They took into account a wind from the corona, which can affect the general solution. The effect of incorporation of the wind losses also depends on viscosity. Taking into account these results, it is apparent that the dependence of their model on viscosity is intermediate between the ADAF and our evaporation model $\mathrm{C}$.

A change in the viscosity parameter results in essentially a parallel shift of a line, without significant change of the line slope. For example, increasing the viscosity parameter $\alpha$ results in moving the limiting line given by the model $\mathrm{C}$ closer to the data points but generally either some of the objects with the broadest lines are already above the threshold or there is a large region to the left of the line which should be populated by broad line objects but no data points are actually there. The steeper relation coming from ADAF seems to better represent the populated region. The best value of the viscosity parameter is around 0.04, as adopted in Fig. 5.

Similarly, we can assess the dependence of the line width on the black hole mass. In Fig. 6 we plot the objects from our sample. Objects occupy predominantly the lower right corner of the diagram. The allowed region, according to a specific model, is shown with lines, as in Fig. 5. Limits for the line width in both models depend on the mass (objects with larger masses are allowed to have broader lines) but the exact shape of this dependence is different in the ADAF model and in the evaporation model. Changing the viscosity parameter $\alpha$ it is possible to move the lines up or down but without a strong change of the shape. Again it is apparent that the region populated by the objects from Table 1 is better described by the 
classical ADAF than a more complex evaporation model, and the favored viscosity parameter $\alpha=0.04$.

\section{Discussion}

Results presented in this paper support the existence of a connection between the BLR and the survival of the cold disk sufficiently close to the central black hole. This agrees with general conclusions of Nicastro et al. (2003) and Laor (2003b), but it takes this idea one step further.

Each specific mechanism of cold disk disruption/ evaporation gives a specific shape of the allowed region for broad line objects in the line width-Eddington ratio and line width-black hole mass diagram. We considered three models of the inner cold disk disappearance and for two of them (classical ADAF and generalized disk evaporation model of Różańska \& Czerny 2000b) we constructed the detailed theoretical region boundaries for the broad line region objects in these two diagrams. We placed on this diagram a large sample of broad line AGN taken from the literature.

Perhaps surprisingly, the classical ADAF model represented the disk disappearance conditions better than the disk evaporation model. The value of the viscosity best representing the allowed parameter space $(\alpha=0.04)$ is quite reasonable, in view of estimates based on AGN variability ( $\sim 0.02$, Starling et al. 2004) or on the results the MHD simulations of the MRI (magneto-rotational) instability now accepted as the physical source of viscosity (again, $~ 0.02$, Winters et al. 2003). However, the apparent superiority of the ADAF solution may be caused by selection effects. The avoidance region of broad line objects is characterized by small black hole masses and small Eddington ratio so these objects are actually very faint.

A consistent picture of an AGN activity emerges from recent papers, including our results. The key parameter governing the character of activity is indeed the Eddington ratio of an object. Specifically, the accretion flow in sources radiating above a per cent, or a few per cent, of the Eddington ratio proceeds predominantly in the form of a cold disk even quite close to the black hole. The continuum spectra of these sources show a prominent optical-UV Big Blue Bump, extending in some sources well into soft X-rays. The optical/UV emission lines are broad, and the iron $\mathrm{K}_{\alpha}$ line is broadened (although not necessarily extremely broad). A natural conclusion is that all those features are related to the existence of the cold disk down to a few hundred - a few tens of the Schwarzschild radii, or less.

Accretion flow in sources radiating at the Eddington ratio below one per cent, or a few per cent of the Eddington ratio do not develop features such as a Big Blue Bump and broad emission lines (Tran 2001, 2003). At the same time they are unlikely to contain radiatively efficient inner flow (e.g. Merloni et al. 2003), and their emission may originate predominantly as a synchrotron emission of the jet (Merloni et al. 2003).

The properties are not just bi-modal but there is also clear effect of the Eddington ratio on all source properties even within its class, and perhaps a smooth transition between them. Many studies show a tight correlation of source properties with a single parameter (Boroson \& Green eigenvector 1, Boroson \& Green 1992; see also Marziani et al. 2003, and the references therein) most probably representing the Eddington ratio.

As for the conclusion that the classical ADAF solution better represents the broad line objects, we treat this result as indicative rather than a proof of the failure of the other models since there are several issues which might have affected the analysis.

\subsection{Accuracy of the theoretical relations}

\subsubsection{The size of the BLR}

In the present paper we adopted the scaling from Kaspi et al. (2000), with $R_{\mathrm{BLR}} \propto \lambda F_{\lambda}(5100 \AA)^{0.7}$. Extensive 20 -year studies of NGC 5548 (Peterson et al. 2002) show that the situation is more complex due to the dependence of the shape of the spectrum on its luminosity in this object. Therefore, two good representations of the source behaviour were found: $R_{\mathrm{BLR}} \propto \lambda F_{\lambda}(5100 \AA)^{0.95}$ and $R_{\mathrm{BLR}} \propto \lambda F_{\lambda}(1350 \AA)^{0.53}$. The Kaspi et al. (2000) formula has an index intermediate between an optical and a UV law since perhaps statistically most objects show weaker dependence of the spectral shape on luminosity than NGC 5548. Since physically we expect a dependence of the BLR size on the ionizing flux (and not total bolometric luminosity) it is likely more appropriate to use UV flux, whenever possible, and perhaps a slightly smaller index in sizeluminosity scaling, if statistically sound scaling is available.

\subsubsection{Evaporation radius and the accretion flow efficiency}

The version of solutions adopted in our considerations are the most basic and simple parametric dependences, either derived from analytical solutions or from analytical crude approximations to the numerical results. A more careful approach does not seem necessary at this stage since the physical assumptions behind the discussed models can be questioned. Therefore, the best support for the model came from as many observational tests as possible.

Liu \& Meyer-Hofmeister (2001) compared the predictions of their model with the constraints for an inner disk radius from spectra analysis and they found a satisfactory agreement for most objects, but Low Luminosity AGN as for instance NGC 4579 (see also Sect. 5.2.4) and M 81 posed a problem for this paper. However, the effect of magnetic field by Meyer \& Meyer-Hofmeister (2002) allowed for its solution.

\subsection{Accuracy of the observational results}

\subsubsection{Intermediate line region}

From careful studies of the UV quasar spectra, Brotherton et al. (1994b) suggested that the traditional BLR consists of two components: one of width $\sim 2000 \mathrm{~km} \mathrm{~s}^{-1}$ and another very broad component of width $\sim 7000 \mathrm{~km} \mathrm{~s}^{-1}$. These components also differ with respect to the net shift in comparison to the 
systemic redshift. Brotherton et al. (1994b) suggested, that the first component forms an Intermediate Line Region (ILR) located further out and possessing a lower medium density and covering factor than the second component, forming a Very Broad Line Region. Similar ideas were suggested earlier by Baldwin et al. (1988) and Francis et al. (1992). As is apparent from the profile of the MgII line in the composite spectra obtained from SDSS (Fig. 5 of Richards et al. 2002), this line might well consist of a narrower core and broader shoulders.

Following those ideas, we assume that the Very Broad Line Region is related to the cold accretion disk, as the component originating closer in and originating in material moving at a faster speed. In the analysis of MgII lines by Forster et al. (2001) and by Kuraszkiewicz et al. $(2002,2004)$ both components were identified, and we used the FWHM of the VBLR component in our analysis. Such differentiation was not always done for other sources used in our paper which resulted in underestimation of some line widths.

\subsection{2. $\mathrm{Mgll}$ vs. $\mathrm{H}_{\beta}$ lines}

Both MgII and $\mathrm{H}_{\beta}$ lines are Low Ionization Lines (LIL) and therefore are supposed to arise from the vicinity of accretion disks (Collin-Souffrin et al. 1988). However, it does not need to mean automatically that they form at the same distance from the central region. For instance, the time delay measured for MgII lines in NGC 5548 (Clavel et al. 1992) is larger by a factor of three than the time delay of $\mathrm{H}_{\beta}$ in the same source (Peterson et al. 1999). The scaling law of Kaspi et al. (2000) (see Eq. (1)) is based on $\mathrm{H}_{\beta}$ and the use of $\mathrm{MgII}$ lines in principle requires an adjustment of the approach. However, McLure \& Jarvis (2002) find the BLR scaling $R_{\mathrm{BLR}} \propto \lambda L_{\lambda}$, if MgII lines are used and the continuum is measured at $3000 \AA$ but otherwise they do not find any systematic difference in $F W H M$ of $\mathrm{MgII}$ and $\mathrm{H}_{\beta}$, as expected if both lines are emitted at the same distance from the central ionizing source.

Among the objects included in Table 1 there are two sources with independent measurements of $\mathrm{MgII}$ and $\mathrm{H}_{\beta}$ by various authors. The measured MgII line is much broader than $\mathrm{H}_{\beta}$ (PG 12111+143: $5400 \mathrm{~km} \mathrm{~s}^{-1}$ vs. $1832 \mathrm{~km} \mathrm{~s}^{-1}$; Mkn 335: $5300 \mathrm{~km} \mathrm{~s}^{-1}$ vs. $1620 \mathrm{~km} \mathrm{~s}^{-1}$ ). However, McLure \& Jarvis give $2050 \mathrm{~km} \mathrm{~s}^{-1}$ for the MgII FWHM of PG $1211+143$. This difference illustrates the possible problems. On the one hand, the data of McLure \& Jarvis (2002) taken with IUE is of lower quality than the FOS/HST data analyzed by Kuraszkiewicz et al. $(2002,2004)$ and no differentiation between the ILR and VBLR components could have been done by those authors. The Kuraszkiewicz et al. data were obtained with the HST, with good S/N. On the other hand, the modeling of MgII in NLS1 galaxies is possibly a subject of large systematic errors due to the presence of strong FeII emission. A specific template has to be used in order to account for FeII before MgII modeling can be done (I Zw1 has been used by Kuraszkiewicz et al., following the analysis of Vestergaard \& Wilkes 2001) and therefore the measurement of the FWHM of MgII may depend on this choice.

\subsubsection{Estimate of Eddington ratio}

The estimate of the Eddington ratio in a given object relies both on estimates of the source bolometric luminosity as well as the black hole mass. The method used in our paper is the simplest but relies on a single measured parameter $\left(\lambda F_{\lambda}\right.$ at some wavelength). Still, errors do not appear large if we compare for example the mass of the black hole determined in this way with masses from BLR monitoring. For example, logarithms of black hole masses for NGC 3227 and NGC 3516 carefully determined by Onken et al. (2003), equal to 7.56 and 7.23 respectively, are similar to the values used in our paper (see Table 1). However, in principle the use of a direct method of a black hole mass estimation would be more appropriate since the mass and the accretion are independent parameters, as stressed in the study of Woo \& Urry (2002).

\subsubsection{Low luminosity AGN}

Low Luminosity AGN do not seem to pose a problem for the picture. Our sample contains a few such objects. The well studied case of NGC 4579 is a good example. Classified as a LINER, this object shows a broad component in its $\mathrm{H}_{\alpha}$ line (Barth et al. 2001) with FWZI of $18000 \mathrm{~km} \mathrm{~s}^{-1}$, and the $F W H M$ can be roughly estimated as $\sim 8000 \mathrm{~km} \mathrm{~s}^{-1}$ (see their Fig. 1). We have this object in our sample (see Table 1), with the FWHM of the MgII line of $6800 \mathrm{~km} \mathrm{~s}^{-1}$. The mass estimate $\left(10^{6} M_{\odot}\right.$ in our paper; $4 \times 10^{6} M_{\odot}$ suggested by Barth et al. 1996) locates this object relatively far to the left in our diagrams (black filled triangle), but still within the allowed region for broad line objects. However, if the mass is actually much larger (Barth et al. 2001 suggest a mass an order of magnitude higher based on black hole mass - bulge velocity dispersion relation) the object will move outside the allowed parameter space as defined by the ADAF model. However, it will remain consistent with a disk evaporation model. Iron line analysis in this source indicates, however, that the standard disk is present in this source and the source radiates at the Eddington ratio of $2 \times 10^{-3}$ (Terashima et al. 2000), only by a factor of 2 lower than obtained in our analysis. Therefore, studies of more low luminosity objects with good mass determination would be particularly valuable.

\subsection{Other samples/objects}

\subsubsection{Low luminosity objects from the SDSS survey}

In the low luminosity broad/double peak $\mathrm{H}_{\alpha}$ sample from SDSS analyzed by Strateva et al. (2003), the tail of the distribution extends up to $\sim 16000 \mathrm{~km} \mathrm{~s}^{-1}$. These objects cannot be included in our study and analyzed in the same manner as the other objects since these AGN usually do not show a Big Blue Bump so Eq. (20) does not apply. However, the measured width of the lines can be compared with constraints presented in Fig. 4. The maximum value of the $F W H M$ of $16000 \mathrm{~km} \mathrm{~s}^{-1}$ indicates that, according to the standard ADAF and a black hole mass $M>10^{8} M_{\odot}$ (Model A, see Fig. 4) the corresponding value of the viscosity parameter $\alpha$ is about 0.02 . If we adopt model B with $\alpha=0.3$ we obtain a strong limit for the strength of the 
magnetic field: the parameter $\beta$ (gas pressure to total pressure ratio) must be larger than 0.7 , i.e. the plasma must be gas pressure dominated. The constraints from model $\mathrm{C}$ give the minimum value of the viscosity $\alpha$ as a function of $\beta$ : $\alpha$ about 0.1 for gas pressure dominated plasma and that could be lower if the magnetic field is important.

\subsubsection{Objects considered by Nicastro et al. (2003)}

The upper limits for the Eddington ratio in Broad line objects, 0.001 , obtained by Nicastro et al. (2003) locate them in Figs. 5 and 6 possibly somewhere between the limits obtained from ADAF and from disk evaporation. However, the measurements of the FWHM of the hidden BLR was not provided by Tran (2001) who conducted the spectro-polarimetric measurements for the sample discussed by Nicastro et al. (2003).

\section{Conclusions}

Tran (2001, 2003) and Laor (2003b) argue that in a fraction of AGN, broad lines are not detected - but not because they are hidden from our line of sight, but because those objects do not develop a BLR. Recent X-ray observation of NGC 6251 supports this view (Gliozzi et al. 2004).

Our study shows that the existence of this class of objects is a natural consequence of the evaporation of the cold disk close to a black hole and the transition of the accretion flow into a hot optically thin phase, if the BLR formation is intimately connected with the cold disk. The transitions happens far away for low Eddington ratio objects, at distances where the ionization conditions would be correct for broad line formation, and the BLR apparently lose the needed disk "support".

Quantitatively, the allowed parameter space (in $\log L / L_{\text {Edd }}$ vs. $\log F W H M$ and $\log M$ vs. $\log$ FWHM; see Figs. 5 and 6) for broad line objects in our sample seems to be better represented by the strong $A D A F$ principle than by the more physical disk evaporation model.

Acknowledgements. We are grateful to Michał Jaroszyński, Jean-Pierre Lasota and Zbyszek Loska for very helpful discussions, and to Greg Madejski for comments to the manuscript. This work was supported in part by grant 2P03D 00322 of the Polish State Committee for Scientific Research (KBN).

\section{References}

Abramowicz, M. A., Chen, X., Kato, S., Lasota, J.-P., \& Regev, O. 1995, ApJ, 438, L37

Baldwin, J. A., McMahon, R., Hazard, C., et al. 1988, ApJ, 327, 103

Barth, A. J., Reichert, G. A., Filippenko, A. V., et al. 1996, AJ, 112, 1829

Barth, A. J., Ho, L. C., Filippenko, A. V., et al. 2001, ApJ, 546, 205

Boroson, T. A., \& Green, R. F. 1992, ApJS, 80, 109

Brotherton, M. S., Wills, B. J., Steidel, C. C., \& Sargent, W. L. W. 1994a, ApJ, 423, 131

Brotherton, M. S., Wills, B. J., Francis, P. J., \& Steidel, C. C. 1994b, ApJ, 430, 495

Chen, K., \& Halpern, J. P. 1989, ApJ, 344, 115
Clavel, J., Nandra, K., Makino, F., et al. 1992, ApJ, 393, 113

Collin, S., Abrassart, A., Czerny, B., Dumont, A.-M., \& Mouchet, M. 2001, EAS Publ. Ser., Vol. 1. Active galactic nuclei in their cosmic environment. JENAM 99, Toulouse, France, September 7-9, 1999, ed. B. Rocca-Volmerange, \& H. Sol (Les Ulis: EDP Sciences), 35

Collin, S., Boisson, C., Mouchet, M., et al. 2002, A\&A, 388, 771

Collin-Souffrin, S., Dyson, J. E., McDowell, J. C., \& Perry, J. J. 1988, MNRAS, 232, 539

Czerny, B. 2003, Active Galactic Nuclei: from Central Engine to Host Galaxy, meeting held in Meudon, France, July 23-27, 2002, ed. S. Collin, F. Combes, \& I. Shlosman (Astronomical Society of the Pacific), ASP Conf. Ser., 290, 59

Czerny, B., Nikołajuk, M., Piasecki, M., \& Kuraszkiewicz, J. 2001, MNRAS, 325, 865

Dultzin-Hacyan, D., Marziani, P., \& Sulentic, J. W. 2000, in Astrophysical Plasmas: Codes, Models, and Observations, Proc. of the conference held in Mexico City, October 25-29, 1999, ed. J. Arthur, N. Brickhouse, \& J. Franco (Ser. Conf.), Rev. Mex. Astron. Astrofis., 9, 308

Elvis, M. 2000, ApJ, 545, 63

Forster, K., Green, P. J., Aldcroft, T., Vestergaard, M., \& Foltz, C. B. 2001, ApJS, 134, 35

Francis, P. J., Hewett, P. C., Foltz, C. B., et al. 1992, ApJ, 398, 476

Haardt, F., Maraschi, L., \& Ghisellini, G. 1994, ApJ, 432, L95

Galeev, A. A., Rosner, R., \& Vaiana, G. S. 1979, ApJ, 229, 318

Honma, F. 1996, PASJ, 48, 77

Ichimaru, S. 1977, ApJ, 214, 840

Janiuk, A., Czerny, B., \& Madejski, G. M. 2001, ApJ, 557, 408

Kaspi, S., Smith, P. S., Netzer, H., et al. 2000, ApJ, 533, 631

Kato, S., \& Nakamura, K. E. 1998, PASJ, 50, 559

Kuraszkiewicz, J. K., Green, P. J., Forster, K., et al. 2002, ApJS, 143, 257

Kuraszkiewicz, J. K., Green, P. J., Crenshaw, D. M., et al. 2004, ApJS, 150,165

Kurpiewski, A., \& Jaroszyński, M. 2000, Acta Astron., 50, 79

Laor, A. 2003a, in AGN Physiscs with the Sloan Digital Sky Survey, ASP Conf. Ser., ed. G. T. Richards, \& P. B. Hall [arXiv: astro-ph/0312415]

Laor, A. 2003b, ApJ, 590, 86

Liu, B. F., Yuan, W., Meyer, F., Meyer-Hofmeister, E., \& Xie, G. Z. 1999, ApJ, 527, L17

Liu, B. F., \& Meyer-Hofmesiter, E. 2001, A\&A, 372, 386

Liu, B. F., Mineshige, S., Meyer, F., Meyer-Hofmeister, E., \& Kawaguchi, T. 2002, ApJ, 575, 117

Marziani, P., Zamanov, K., Sulentic, J. W., \& Calvani, M. 2003, MNRAS, 345, 1133

McLure, R. J., \& Jarvis, M. J. 2002, MNRAS, 337, 109

Merloni, A., Heinz, S., \& di Matteo, T. 2003, MNRAS, 345, 1057

Merloni, A., Heinz, S., \& Sunyaev, R. A. 2003 [arXiv: astro-ph/0305261]

Meyer, F., \& Meyer-Hofmeister, E. 1994, A\&A, 288, 175

Meyer, F., \& Meyer-Hofmeister, E. 2002, A\&A, 392, L5

Meyer-Hofmeister, E., \& Meyer, F. 1999, A\&A, 348, 154

Meyer-Hofmeister, E., \& Meyer, F. 2003, A\&A, 402, 1013

Narayan, R., Mahadevan, R., \& Quataert, E. 1998, in The Theory of Black Hole Accretion Disks, ed. M. A. Abramowicz, G. Bjornsson, \& J. E. Pringle (Cambridge: Cambridge Univ. Press), 148

Narayan, R., \& Yi, I. 1994, ApJ, 428, L13

Nicastro, F., Martocchia, A., \& Matt, G. 2003, ApJ, 589, L13

O'Brien, et al. 2003, in XEUS - studying the evolution of the hot universe, ed. G. Hasinger, Th. Boller, \& A. N. Parmer, MPE Report, 281,191 
Onken, C. A., Peterson, B. M., Dietrich, M., Robinson, A., \& Salamanca, I. M. 2003, ApJ, 285, 121

Peterson, B. M., \& Wandel, A. 1999, ApJ, 521, L95

Peterson, B. M., Barth, A. J., Berlind, P., et al. 1999, ApJ, 510, 659

Pounds, K. A., Reeves, J. N., King, A. R., et al. 2003, MNRAS, 345, 705

Richards, G. T., Vanden Berk, D. E., Reichard, T. A., et al. 2002, AJ, 124,1

Różańska, A., \& Czerny, B. 2000a, MNRAS, 316, 473

Różańska, A., \& Czerny, B. 2000b, A\&A, 360, 1170

Różańska, A., Czerny, B., Siemiginowska, A., Dumont, A.-M., \& Kawaguchi, T. 2004, ApJ, 600, 96

Sako, M., Kahn, S. M., Branduardi-Raymont, G., et al. 2003, ApJ, 596,114
Shang, Z., Wills, B. J., Robinson, E. L., et al. 2003, ApJ, 586, 52

Starling, R. L. C., Siemiginowska, A., Uttley, P., \& Soria, R. 2004, MNRAS, 347, 67

Steidel, C. C., \& Sargent, W. L. W. 1991, ApJ, 382, 433

Strateva, I. V., Strauss, M. A., Hao, L., et al. 2003, AJ, 126, 1720

Terashima, Y., Ho, L. C., Ptak, A. F., et al. 2000, ApJ, 535, L79

Tran, H. D. 2001, ApJ, 554, L19

Tran, H. D. 2003, ApJ, 583, 632

Turolla, R., \& Dullemond, C. P. 2000, ApJ, 531, L49

Vestergaard, M., \& Wilkes, B. J. 2001, ApJS, 134, 1

Wandel, A., Peterson, B. M., \& Malkan, M. A. 1999, ApJ, 526, 579

Winters, W. F., Balbus, S. A., \& Hawley, J. F. 2003, MNRAS, 340, 519

Woo, J.-H., \& Urry, C. M. 2002, ApJ, 579, 530 


\section{Online Material}




\section{Appendix A: Disk-corona mass exchange in the corona model with magnetic pressure component}

Disk evaporation due to conductive heating of the disk interior by the hot accreting corona was proposed by Meyer \& Meyer-Hofmeister (1994) in the context of the cataclysmic variables. This approach was further generalized in a number od papers (Meyer-Hofmeister \& Meyer 1999; Turolla \& Dullemond 2000; Różańska \& Czerny 2000a). The evaporation rate is calculated on the basis of the computations of the conduction flux between the disk and the corona. The effect depends both on the description of the conduction and the description of the corona heating/cooling and a detailed approach to its vertical structure. In accreting corona models discussed in this context, the standard assumption was that the heating is described by the viscosity parameter $\alpha$ and the gas pressure in the corona, as in the description of the purely hot (ADAF) flows. In the context of ADAF a generalization is frequently made to include the magnetic pressure in addition to the disk pressure, since in those models the magnetic field is needed to provide synchrotron photons for the Compton cooling. In corona models, the synchrotron photons are relatively unimportant since the soft disk photons are abundant but nevertheless the magnetic component of the total pressure can also be present. Such an effect was recently included in their model by Meyer \& Meyer-Hofmeister (2002).

Here we generalize the model developed by Różańska \& Czerny (2000b) in order to include this additional magnetic pressure component.

We describe the local disk vertical structure as in Różańska \& Czerny (2000b), Sect. 2.2, but we assume now a more general formula for the pressure, $P$ :

$P=\frac{k}{m_{\mathrm{H}} \rho T_{i}}+P_{m}=\frac{1}{\beta} \frac{k}{m_{\mathrm{H}} \rho T_{i}}$,

where $\beta$ is the gas pressure to the total (gas + magnetic) pressure ratio. Gas pressure includes only the ion component, as before.

We determine the local disk evaporation rate $\dot{m}_{z}$ as a function of the total (disk + corona) accretion rate, coronal accretion rate, the black hole mass and the parameters $\alpha$ (viscosity) and $\beta$ (magnetic field) from the Eq. (15) of Różańska \& Czerny (2000b). Modifying the code we found a numerical error in the program. New results for $\beta=1$ (no magnetic pressure) and $\alpha=01$ are shown in Fig. A.1. The dependence on the viscosity is now much weaker than in Różańska \& Czerny (2000b).

The local evaporation rate shown in Fig. A.1 increases with a decrease of the viscosity parameter and decreases with an increase of the magnetic field contribution to the pressure. These trends can be understood by considering the relative contributions to the total pressure. Specifically, the total pressure at the base of the corona is determined by the coronal accretion rate under consideration and the viscosity $\alpha$. The gas pressure there is thus proportional to $\beta / \alpha$. Since the ion temperature is not significantly lower than the virial temperature in the coronal part of the flow, the density is mostly determined by the pressure. The electron temperature is given by the equilibrium be-

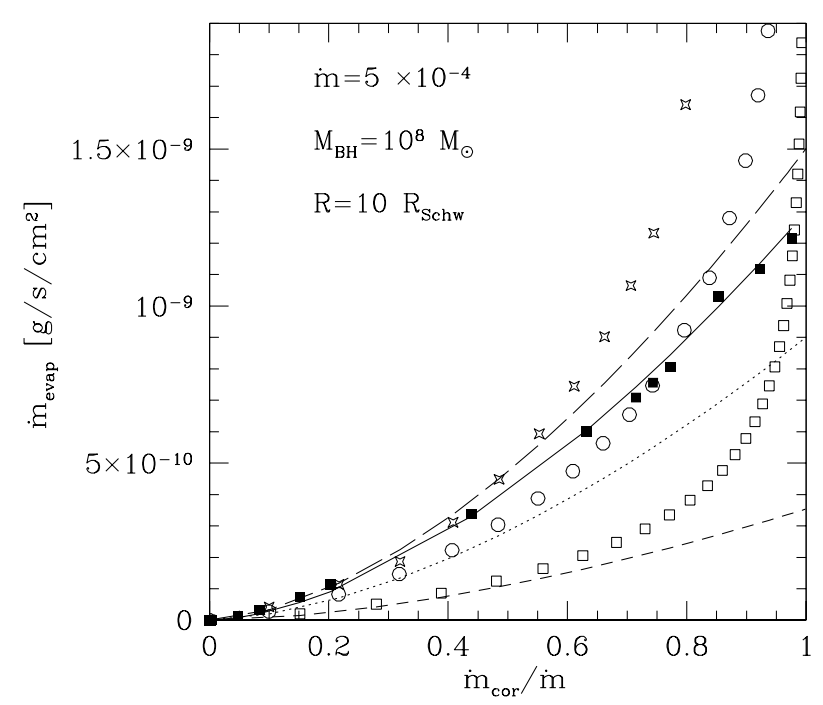

Fig. A.1. The local relation between the accretion rate in the corona and the evaporation rate of the disk at $10 R_{\mathrm{Schw}}$ for the total accretion rate $\dot{m}=5 \times 10^{-4}$ and black hole mass $10^{8} M_{\odot}$. Filled squares are from Różańska \& Czerny (2000), open circles are new results for $\alpha=0.1$, $\beta=1$, open squares are for $\alpha=0.1, \beta=0.5$ and stars are for $\alpha=$ $0.05, \beta=1$.

tween the electron heating and cooling, and it increases with the rise in the density. Therefore, the electron temperature increases with $\beta$ and decreases with $\alpha$. This trend determines the trend of the integral determining $\dot{m}_{z}$ (Eq. (15) of Różańska \& Czerny 2000).

Numerical solutions can be roughly represented by the analytical formula:

$\dot{m}_{\text {evap }}=1.64 \times 10^{-3} \alpha_{0.1}^{-1} \beta^{1.35} M_{8}^{-1} \dot{m}_{\text {cor }}^{5 / 3} R^{-3 / 4}$.

This formula combined with the need for the mass conservation in the flow (total accretion rate $=$ coronal accretion rate + disk accetion rate) gives the solution for the dependence of the coronal accretion rate on the radius:

$\dot{m}_{\text {cor }}=2.62 \times 10^{2} \alpha_{0.1}^{3 / 2} \beta^{-2.025} R^{-15 / 8}$.

The condition $\dot{m}_{\text {cor }}=\dot{m}$ determines the evaporation radius (Eq. (11) in this paper). Here we neglected the issue of the coronal condensation in the innermost region, discussed by Różańska \& Czerny (2000). 
B. Czerny et al.: Disk evaporation and the BLR, Online Material p 3

Table 1. Broad line objects: measured line widths and luminosities, estimates of central black holes masses and Eddington ratios, as described in the text, and the references.

\begin{tabular}{|c|c|c|c|c|c|c|c|}
\hline Name & Spectrum & Redshift & $F W H M(\mathrm{MgII})$ & $F_{\lambda}(2800 \AA)$ & $\log M$ & $\log L / L_{\mathrm{Edd}}$ & Ref. \\
\hline LBQS 0010+0146 & $0010+01461 a$ & 0.587 & 13000. & -15.3856 & 9.72 & -1.58 & 1 \\
\hline LBQS 0018-0252 & 0018-0252la & 0.618 & 5600. & -15.4883 & 8.96 & -0.86 & 1 \\
\hline LBQS 0022-0140 & 0022-01401a & 0.776 & 8500. & -15.4825 & 9.51 & -1.14 & 1 \\
\hline LBQS 0028-0101 & 0028-0101la & 0.543 & 12000. & -15.8062 & 9.29 & -1.67 & 1 \\
\hline LBQS 0042-2750 & 0042-2750la & 0.741 & 6400. & -15.7581 & 9.04 & -0.99 & 1 \\
\hline LBQS 0047-3059 & 0047-3059la & 0.559 & 8000. & -14.5679 & 9.83 & -0.93 & 1 \\
\hline LBQS 0048-0133 & 0048-01331a & 0.763 & 8500. & -14.5792 & 10.13 & -0.88 & 1 \\
\hline LBQS 0055-2948 & 0055-2948la & 0.663 & 3940. & -15.7641 & 8.52 & -0.61 & 1 \\
\hline LBQS 0056-0009 & 0056-0009la & 0.717 & 7000. & -14.7753 & 9.77 & -0.79 & 1 \\
\hline LBQS 0057+0000 & $0057+00001 \mathrm{a}$ & 0.776 & 8000. & -14.7906 & 9.95 & -0.88 & 1 \\
\hline LBQS 0102-2713 & 0102-27131a & 0.780 & 5400. & -15.5035 & 9.11 & -0.75 & 1 \\
\hline LBQS 0102-0147 & 0102-0147la & 0.571 & 10000. & -14.9600 & 9.76 & -1.24 & 1 \\
\hline LBQS 0103-2622 & 0103-2622la & 0.776 & 7000. & -15.4250 & 9.39 & -0.95 & 1 \\
\hline LBQS 0107-0235 & 0107-0235la & 0.958 & 10500. & -15.3280 & 9.99 & -1.20 & 1 \\
\hline LBQS 0252+0141 & $0252+01411 \mathrm{a}$ & 0.621 & 13000. & -15.1197 & 9.95 & -1.48 & 1 \\
\hline LBQS 0253-0138 & 0253-0138la & 0.879 & 16500. & -14.9133 & 10.60 & -1.50 & 1 \\
\hline LBQS 0257+0025 & $0257+00251 \mathrm{a}$ & 0.532 & 6400. & -14.7526 & 9.46 & -0.81 & 1 \\
\hline LBQS 0301+0010 & $0301+00101 \mathrm{a}$ & 0.638 & 6600. & -14.7610 & 9.63 & -0.78 & 1 \\
\hline LBQS 0303-0132 & 0303-0132la & 0.606 & 6500. & -14.7173 & 9.61 & -0.77 & 1 \\
\hline LBQS 0303-0241 & 0303-0241la & 0.686 & 5000. & -15.0694 & 9.24 & -0.60 & 1 \\
\hline LBQS $0305+0222$ & $0305+02221 \mathrm{a}$ & 0.590 & 10400. & -14.8150 & 9.93 & -1.21 & 1 \\
\hline LBQS 0307-0015 & 0307-0015la & 0.770 & 6400. & -14.8683 & 9.69 & -0.71 & 1 \\
\hline LBQS 1027-0149 & 1027-0149la & 0.754 & 11500. & -15.5531 & 9.70 & -1.43 & 1 \\
\hline LBQS $1137+0051$ & $1137+00511 \mathrm{a}$ & 0.874 & 9500. & -15.8626 & 9.45 & -1.31 & 1 \\
\hline LBQS $1138+0003$ & $1138+00031 \mathrm{a}$ & 0.500 & 14000. & -15.4346 & 9.61 & -1.72 & 1 \\
\hline LBQS $1205+1729$ & $1205+17291 \mathrm{a}$ & 0.548 & 16000. & -15.0968 & 10.04 & -1.70 & 1 \\
\hline LBQS $1211+0841$ & $1211+08411 \mathrm{a}$ & 0.585 & 8800. & -15.3781 & 9.38 & -1.24 & 1 \\
\hline LBQS $1222+1640$ & $1222+16401 \mathrm{a}$ & 0.549 & 9600. & -15.0785 & 9.61 & -1.25 & 1 \\
\hline LBQS $1222+0901$ & $1222+09011 \mathrm{a}$ & 0.535 & 13000. & -13.4831 & 10.97 & -1.04 & 1 \\
\hline LBQS 1224+1604 & 1224+1604la & 0.533 & 7500. & -15.6004 & 9.01 & -1.20 & 1 \\
\hline LBQS $1236+1802$ & $1236+18021 \mathrm{a}$ & 0.517 & 14000. & -15.4997 & 9.60 & -1.72 & 1 \\
\hline LBQS $1237+0950$ & $1237+09501 \mathrm{a}$ & 0.736 & 11000. & -15.1948 & 9.89 & -1.30 & 1 \\
\hline LBQS $1240+0224$ & $1240+02241 \mathrm{a}$ & 0.790 & 13000. & -15.3358 & 10.00 & -1.46 & 1 \\
\hline LBQS $1240+1745$ & $1240+17451 \mathrm{a}$ & 0.549 & 11500. & -14.9485 & 9.86 & -1.37 & 1 \\
\hline LBQS $1243+1456$ & $1243+14561 \mathrm{a}$ & 0.582 & 10500. & -15.3523 & 9.55 & -1.39 & 1 \\
\hline LBQS $1244+1329$ & $1244+13291 \mathrm{a}$ & 0.512 & 2600. & -14.7649 & 8.64 & -0.05 & 1 \\
\hline LBQS $1245+1719$ & $1245+17191 \mathrm{a}$ & 0.752 & 10000. & -15.2126 & 9.82 & -1.21 & 1 \\
\hline LBQS $1250+0109$ & $1250+01091 \mathrm{a}$ & 0.792 & 13200. & -15.6707 & 9.78 & -1.57 & 1 \\
\hline LBQS 1313-0228 & 1313-0228la & 0.704 & 5200. & -15.6822 & 8.86 & -0.81 & 1 \\
\hline LBQS $1315+0150$ & $1315+01501 \mathrm{a}$ & 0.539 & 12500. & -15.0981 & 9.81 & -1.49 & 1 \\
\hline LBQS 1319+0033 & $1319+00331 \mathrm{a}$ & 0.530 & 11400 . & -15.2236 & 9.63 & -1.45 & 1 \\
\hline LBQS 1322-0204 & $1322-02041 \mathrm{a}$ & 0.573 & 7800. & -14.9842 & 9.53 & -1.03 & 1 \\
\hline LBQS $1323+0205$ & $1323+02051 \mathrm{a}$ & 0.641 & 4400. & -15.4965 & 8.77 & -0.64 & 1 \\
\hline LBQS $1326+0206$ & $1326+02061 \mathrm{a}$ & 1.442 & 13000. & -15.1133 & 10.70 & -1.16 & 1 \\
\hline LBQS 1326-0257 & 1326-0257la & 0.743 & 5000. & -15.3303 & 9.12 & -0.65 & 1 \\
\hline LBQS 1328+0205 & $1328+02051 \mathrm{a}$ & 0.692 & 3500. & -14.3684 & 9.43 & -0.08 & 1 \\
\hline LBQS 1340-0020 & 1340-0020la & 0.786 & 11000. & -15.6413 & 9.64 & -1.41 & 1 \\
\hline LBQS $1433+0011$ & $1433+00111 \mathrm{a}$ & 0.583 & 9000. & -15.4353 & 9.36 & -1.28 & 1 \\
\hline LBQS 1440-0234 & 1440-0234la & 0.678 & 4400. & -14.2734 & 9.67 & -0.26 & 1 \\
\hline LBQS $1445+0222$ & $1445+02221 \mathrm{a}$ & 0.775 & 6800. & -15.3117 & 9.44 & -0.90 & 1 \\
\hline
\end{tabular}


Table 1. continued.

\begin{tabular}{|c|c|c|c|c|c|c|c|}
\hline Name & Spectrum & Redshift & $F W H M(\mathrm{MgII})$ & $F_{\lambda}(2800 \AA)$ & $\log M$ & $\log L / L_{\mathrm{Edd}}$ & Ref. \\
\hline LBQS 2132-4228 & 2132-4228la & 0.569 & 6500. & -15.9061 & 8.72 & -1.15 & 1 \\
\hline LBQS 2142-4318 & 2142-4318la & 1.118 & 5500. & -16.0346 & 9.07 & -0.79 & 1 \\
\hline LBQS 2154-2105 & 2154-2105la & 0.575 & 6500. & -14.7796 & 9.52 & -0.81 & 1 \\
\hline LBQS 2203-2134 & 2203-2134la & 0.576 & 13500. & -15.7686 & 9.46 & -1.74 & 1 \\
\hline LBQS 2244+0020 & $2244+00201 \mathrm{a}$ & 0.973 & 12500. & -14.7397 & 10.57 & -1.17 & 1 \\
\hline LBQS 2350-0012 & 2350-0012la & 0.561 & 5600. & -14.6781 & 9.44 & -0.65 & 1 \\
\hline LBQS 2353+0032 & $2353+00321 \mathrm{a}$ & 0.558 & 12500. & -15.4269 & 9.61 & -1.58 & 1 \\
\hline \multicolumn{8}{|c|}{ HST/FOS PRE-COSTAR: } \\
\hline III ZW 2 & $0010+1058 \mathrm{ra}$ & 0.089 & 8700. & -14.1559 & 8.86 & -1.45 & 2 \\
\hline I ZW 1 & $0053+1241 \mathrm{ra}$ & 0.061 & 5550. & -13.7892 & 8.48 & -1.06 & 2 \\
\hline Fairall 9 & 0123-5848ra & 0.047 & 5750. & -13.7382 & 8.38 & -1.14 & 2 \\
\hline PKS 0403-13 & 0405-1308ra & 0.571 & 8600. & -14.9150 & 9.66 & -1.09 & 2 \\
\hline 3C 207 & $0840+1312 \mathrm{ra}$ & 0.681 & 9500. & -15.1675 & 9.72 & -1.19 & 2 \\
\hline $3 \mathrm{C} 215$ & 0906+1646ra & 0.412 & 14400 . & -15.2551 & 9.61 & -1.75 & 2 \\
\hline B2 $0923+392$ & $0927+3902 \mathrm{ra}$ & 0.695 & 12000. & -14.6144 & 10.33 & -1.22 & 2 \\
\hline B2 $0923+392$ & $0927+3902 \mathrm{rb}$ & 0.695 & 13900. & -14.8952 & 10.26 & -1.43 & 2 \\
\hline $3 \mathrm{C} 263$ & $1139+6547 \mathrm{ra}$ & 0.646 & 17000. & -14.5002 & 10.65 & -1.51 & 2 \\
\hline PG $1211+143$ & $1214+1403 \mathrm{ra}$ & 0.081 & 5400. & -13.8469 & 8.60 & -0.97 & 2 \\
\hline MRK 205 & $1221+7518 \mathrm{rb}$ & 0.071 & 6850. & -13.9038 & 8.68 & -1.23 & 2 \\
\hline PG $1226+023$ & $1229+0203 \mathrm{ra}$ & 0.158 & 7400. & -13.0137 & 9.90 & -0.80 & 2 \\
\hline 3C 277.1 & $1252+5634 \mathrm{ra}$ & 0.321 & 7100. & -15.0441 & 8.95 & -1.16 & 2 \\
\hline 3C 279 & $1256-0547 \mathrm{ra}$ & 0.536 & 17000. & -14.7935 & 10.29 & -1.67 & 2 \\
\hline NGC 5548 & $1417+2508 \mathrm{ra}$ & 0.017 & 8450. & -14.2071 & 7.75 & -1.89 & 2 \\
\hline PG $1415+451$ & $1417+4456 \mathrm{ra}$ & 0.114 & 4500. & -14.4230 & 8.26 & -0.89 & 2 \\
\hline 3C 334 & $1620+1736 r b$ & 0.555 & 9600. & -14.5985 & 9.96 & -1.10 & 2 \\
\hline 3C 345 & $1642+3948 \mathrm{ra}$ & 0.593 & 10000. & -14.6169 & 10.03 & -1.12 & 2 \\
\hline B2 $2201+315 A$ & $2203+3145 \mathrm{ra}$ & 0.295 & 8200. & -13.9539 & 9.78 & -0.98 & 2 \\
\hline 4C 11.72 & $2254+1136 \mathrm{ra}$ & 0.326 & 9000. & -14.2875 & 9.70 & -1.13 & 2 \\
\hline \multicolumn{8}{|c|}{ HST/FOS POST-COSTAR: } \\
\hline MRK 335 & $0006+2012 \mathrm{oa}$ & 0.026 & 5300. & -13.4173 & 8.16 & -1.14 & 3 \\
\hline PG $0026+129$ & $0029+1316$ ob & 0.142 & 6700. & -13.9626 & 9.08 & -1.03 & 3 \\
\hline MRK 348 & $0048+3157 \mathrm{oa}$ & 0.015 & 2400. & -15.8095 & 5.45 & -1.31 & 3 \\
\hline I ZW 1 & $0053+1241$ oa & 0.061 & 5850. & -13.7184 & 8.57 & -1.08 & 3 \\
\hline 0318-196 & 0320-1926oa & 0.104 & 10000. & -15.7505 & 7.96 & -2.01 & 3 \\
\hline AKN 120 & 0516-0008oa & 0.033 & 9100. & -13.2105 & 8.92 & -1.48 & 3 \\
\hline PG 0947+396 & $0950+39260 a$ & 0.206 & 5300. & -14.3277 & 8.88 & -0.83 & 3 \\
\hline PG $1001+054$ & $1004+0513 \mathrm{oa}$ & 0.161 & 4800. & -14.6082 & 8.42 & -0.90 & 3 \\
\hline B2 $1028+313$ & $1030+3102 \mathrm{oa}$ & 0.178 & 10000. & -14.5558 & 9.17 & -1.49 & 3 \\
\hline ZW 212.025 & $1034+3938$ oa & 0.042 & 3100. & -14.9705 & 6.91 & -1.01 & 3 \\
\hline NGC 3516 & $1106+7234 \mathrm{oa}$ & 0.009 & 6450. & -13.4862 & 7.63 & -1.61 & 3 \\
\hline NGC 3516 & $1106+7234 \mathrm{ob}$ & 0.009 & 6000 & -13.5925 & 7.49 & -1.58 & 3 \\
\hline NGC 3516 & $1106+7234 \mathrm{oc}$ & 0.009 & 6650. & -13.6224 & 7.56 & -1.68 & 3 \\
\hline NGC 3516 & $1106+7234$ od & 0.009 & 6000. & -13.4524 & 7.59 & -1.53 & 3 \\
\hline NGC 3516 & $1106+7234 \mathrm{oe}$ & 0.009 & 7550. & -13.9228 & 7.46 & -1.88 & 3 \\
\hline PG $1114+445$ & $1117+4413$ oa & 0.144 & 7000. & -14.3122 & 8.88 & -1.17 & 3 \\
\hline PG $1114+445$ & $1117+4413 \mathrm{ob}$ & 0.144 & 6600. & -14.4533 & 8.73 & -1.16 & 3 \\
\hline MC $1118+12$ & $1121+1236 \mathrm{oa}$ & 0.685 & 9500. & -15.4223 & 9.55 & -1.26 & 3 \\
\hline GQ COMAE & $1204+2754 \mathrm{oa}$ & 0.165 & 12000. & -14.8342 & 9.08 & -1.76 & 3 \\
\hline
\end{tabular}


B. Czerny et al.: Disk evaporation and the BLR, Online Material p 5

Table 1. continued.

\begin{tabular}{|c|c|c|c|c|c|c|c|}
\hline Name & Spectrum & Redshift & $F W H M(\mathrm{MgII})$ & $F_{\lambda}(2800 \AA)$ & $\log M$ & $\log L / L_{\mathrm{Edd}}$ & Ref \\
\hline $1219+047$ & $1221+0430 \mathrm{ob}$ & 0.094 & 10000. & -15.1540 & 8.31 & -1.86 & 3 \\
\hline QSO1219+057 & $1219+0545$ oа & 0.114 & 3300. & -15.5099 & 7.23 & -0.95 & 3 \\
\hline $1220+1601$ & $1223+1545 \mathrm{ob}$ & 0.081 & 6500. & -16.1004 & 7.18 & -1.81 & 3 \\
\hline NGC 4579 & $1237+1149 \mathrm{oa}$ & 0.005 & 6800. & -15.3764 & 5.99 & -2.38 & 3 \\
\hline PG $1322+659$ & $1323+6541$ oa & 0.168 & 10000. & -14.3634 & 9.26 & -1.45 & 3 \\
\hline MRK 270 & $1341+6740$ oа & 0.009 & 2000 . & -15.9921 & 4.85 & -1.34 & 3 \\
\hline PG $1352+183$ & $1354+1805$ oа & 0.152 & 5200. & -14.3633 & 8.62 & -0.91 & 3 \\
\hline PG $1415+451$ & $1417+4456$ oа & 0.114 & 5900. & -14.3667 & 8.53 & -1.11 & 3 \\
\hline MRK 478 & $1442+35260 \mathrm{a}$ & 0.079 & 6400. & -13.8295 & 8.74 & -1.12 & 3 \\
\hline MR 2251-178 & $2254-1734$ oa & 0.068 & 10950. & -13.9389 & 9.03 & -1.66 & 3 \\
\hline NGC 7469 & $2303+0852 \mathrm{oa}$ & 0.016 & 4200. & -13.5032 & 7.59 & -1.09 & 3 \\
\hline IRAS13349+2438 & $1337+2423 \mathrm{ob}$ & 0.108 & 9300. & -14.7569 & 8.62 & -1.64 & 3 \\
\hline ARP 102B & $1719+4858$ oа & 0.024 & 15500. & -15.2975 & 7.72 & -2.65 & 3 \\
\hline CYGNUS A & $1959+4044$ oa & 0.056 & 11500. & -15.1480 & 8.10 & -2.12 & 3 \\
\hline Name & & & $F W H M\left(\mathrm{H}_{\beta}\right)$ & $\lambda F_{\lambda}(5100 \AA)$ & $\log M$ & $\log L / L_{\mathrm{Edd}}$ & Ref \\
\hline $3 \mathrm{C} 120$ & $\ldots$ & $\ldots$ & 1910. & 0.73000 & 7.36 & -0.64 & 4 \\
\hline $3 C 390.3$ & $\ldots$ & $\ldots$ & 10000. & 0.64000 & 8.53 & -1.87 & 4 \\
\hline Akn 120 & $\ldots$ & $\ldots$ & 5800. & 1.39000 & 8.26 & -1.27 & 4 \\
\hline F9 & $\ldots$ & $\ldots$ & 5780. & 1.37000 & 7.90 & -0.91 & 4 \\
\hline IC 4329A & $\ldots$ & $\ldots$ & 5050. & 0.16400 & 6.70 & -0.63 & 4 \\
\hline Mrk 79 & $\ldots$ & $\ldots$ & 4470. & 0.42300 & 7.72 & -1.24 & 4 \\
\hline Mrk 110 & $\ldots$ & $\ldots$ & 1430. & 0.38000 & 6.75 & -0.31 & 4 \\
\hline Mrk 335 & $\ldots$ & $\ldots$ & 1620. & 0.62200 & 6.80 & -0.15 & 4 \\
\hline Mrk 509 & $\ldots$ & $\ldots$ & 2270 . & 1.47000 & 7.76 & -0.74 & 4 \\
\hline Mrk 590 & $\ldots$ & $\ldots$ & 2470 . & 0.51000 & 7.25 & -0.69 & 4 \\
\hline Mrk 817 & $\ldots$ & $\ldots$ & 4490. & 0.52600 & 7.64 & -1.07 & 4 \\
\hline NGC 3227 & $\ldots$ & $\ldots$ & 4920. & 0.02020 & 7.59 & -2.43 & 4 \\
\hline NGC 3783 & $\ldots$ & $\ldots$ & 3790. & 0.17700 & 6.97 & -0.87 & 4 \\
\hline NGC 4051 & $\ldots$ & $\ldots$ & 1170. & 0.00525 & 6.11 & -1.54 & 4 \\
\hline NGC 4151 & $\ldots$ & $\ldots$ & 5910. & 0.07200 & 7.18 & -1.47 & 4 \\
\hline NGC 5548 & $\ldots$ & $\ldots$ & 6300. & 0.27000 & 8.09 & -1.80 & 4 \\
\hline NGC 7469 & $\ldots$ & $\ldots$ & 3000. & 0.55300 & 6.81 & -0.22 & 4 \\
\hline Name & & & $F W H M\left(\mathrm{H}_{\beta}\right)$ & $\lambda F_{\lambda}(5100 \AA)$ & $\log M$ & $\log L / L_{\mathrm{Edd}}$ & Ref \\
\hline PG 0026+129 & $\ldots$ & $\ldots$ & 2100. & 7.00000 & 7.73 & -0.03 & 5 \\
\hline PG $0052+251$ & $\ldots$ & $\ldots$ & 3990. & 6.50000 & 8.34 & -0.68 & 5 \\
\hline PG 0804+761 & $\ldots$ & $\ldots$ & 2984. & 6.60000 & 8.28 & -0.60 & 5 \\
\hline PG 0844+349 & $\ldots$ & $\ldots$ & 2730 . & 1.72000 & 7.33 & -0.25 & 5 \\
\hline PG $0953+414$ & $\ldots$ & $\ldots$ & 2885. & 11.90000 & 8.26 & -0.34 & 5 \\
\hline PG $1211+143$ & $\ldots$ & $\ldots$ & 1832. & 4.93000 & 7.61 & -0.06 & 5 \\
\hline PG $1226+023$ & $\ldots$ & $\ldots$ & 3416. & 64.40000 & 8.74 & -0.08 & 5 \\
\hline PG $1229+204$ & $\ldots$ & $\ldots$ & 3440. & 0.94000 & 7.88 & -1.05 & 5 \\
\hline PG $1307+085$ & $\ldots$ & $\ldots$ & 4190. & 5.27000 & 8.45 & -0.87 & 5 \\
\hline PG $1351+640$ & $\ldots$ & $\ldots$ & 1404. & 4.38000 & 7.66 & -0.17 & 5 \\
\hline PG $1411+442$ & $\ldots$ & $\ldots$ & 2456. & 3.25000 & 7.90 & -0.54 & 5 \\
\hline PG $1426+015$ & $\ldots$ & $\ldots$ & 6250. & 4.09000 & 8.67 & -1.21 & 5 \\
\hline PG $1613+658$ & $\ldots$ & $\ldots$ & 7000. & 6.69000 & 8.38 & -0.70 & 5 \\
\hline PG $1617+175$ & $\ldots$ & $\ldots$ & 5120. & 2.37000 & 8.44 & -1.21 & 5 \\
\hline PG $1700+518$ & $\ldots$ & $\ldots$ & 2180. & 27.10000 & 7.78 & 0.51 & 5 \\
\hline PG $1704+608$ & $\ldots$ & $\ldots$ & 890. & 35.60000 & 7.57 & 0.84 & 5 \\
\hline PG 2130+099 & $\ldots$ & $\ldots$ & 2410. & 2.16000 & 8.16 & -0.97 & 5 \\
\hline Name & & Redshift & $F W H M\left(\mathrm{H}_{\beta}\right)$ & $\log L_{v}(1549 \AA)$ & $\log M$ & $\log L / L_{\mathrm{Edd}}$ & Ref \\
\hline PG 0947+396 & $\ldots$ & 0.2056 & 4830. & 30.33 & 8.76 & -0.89 & 6 \\
\hline PG $0953+414$ & $\ldots$ & 0.2341 & 3130. & 30.59 & 8.56 & -0.44 & 6 \\
\hline
\end{tabular}


Table 1. continued.

\begin{tabular}{|c|c|c|c|c|c|c|c|}
\hline Name & Spectrum & Redshift & $F W H M(\mathrm{MgII})$ & $F_{\lambda}(2800 \AA)$ & $\log M$ & $\log L / L_{\mathrm{Edd}}$ & Ref. \\
\hline PG 1001+054 & $\ldots$ & 0.1603 & 1740. & 29.73 & 7.45 & -0.19 & 6 \\
\hline PG 1114+445 & $\ldots$ & 0.1440 & 4570. & 29.80 & 8.34 & -1.01 & 6 \\
\hline PG $1115+407$ & $\ldots$ & 0.1541 & 1720. & 30.08 & 7.68 & -0.07 & 6 \\
\hline PG $1116+215$ & $\ldots$ & 0.1759 & 2920. & 30.67 & 8.56 & -0.36 & 6 \\
\hline PG $1202+281$ & $\ldots$ & 0.1651 & 5050. & 29.51 & 8.22 & -1.18 & 6 \\
\hline PG 1216+069 & $\ldots$ & 0.3319 & 5190. & 30.69 & 9.07 & -0.85 & 6 \\
\hline PG $1226+023$ & $\ldots$ & 0.1575 & 3520. & 31.37 & 9.21 & -0.31 & 6 \\
\hline PG $1309+355$ & $\ldots$ & 0.1823 & 2940. & 30.21 & 8.24 & -0.50 & 6 \\
\hline PG $1322+659$ & $\ldots$ & 0.1675 & 2790. & 30.02 & 8.06 & -0.51 & 6 \\
\hline PG $1352+183$ & $\ldots$ & 0.1510 & 3600 . & 30.04 & 8.30 & -0.73 & 6 \\
\hline PG $1402+261$ & $\ldots$ & 0.1650 & 1910. & 30.40 & 8.00 & -0.07 & 6 \\
\hline PG $1411+442$ & $\ldots$ & 0.0895 & 2670. & 29.56 & 7.70 & -0.61 & 6 \\
\hline PG $1415+451$ & $\ldots$ & 0.1143 & 2620. & 29.72 & 7.80 & -0.55 & 6 \\
\hline PG $1425+267$ & $\ldots$ & 0.3637 & 9410. & 30.42 & 9.40 & -1.45 & 6 \\
\hline PG $1427+480$ & $\ldots$ & 0.2203 & 2540. & 30.20 & 8.11 & -0.38 & 6 \\
\hline PG $1440+356$ & $\ldots$ & 0.0773 & 1450. & 29.90 & 7.41 & 0.02 & 6 \\
\hline PG $1444+407$ & $\ldots$ & 0.2670 & 2480 & 30.63 & 8.39 & -0.23 & 6 \\
\hline PG $1512+370$ & $\ldots$ & 0.3700 & 6810. & 30.66 & 9.28 & -1.09 & 6 \\
\hline PG $1543+489$ & $\ldots$ & 0.4000 & 1560. & 30.75 & 8.07 & 0.21 & 6 \\
\hline PG $1626+554$ & $\ldots$ & 0.1317 & 4490. & 30.11 & 8.54 & -0.90 & 6 \\
\hline Name & & Redshift & $F W H M(\mathrm{MgII})$ & $M(2200 \AA)$ & $\log M$ & $\log L / L_{\mathrm{Edd}}$ & Ref. \\
\hline $0009-0138$ & $\ldots$ & 2.0000 & 3500 & -28.0 & 10.04 & 0.24 & 7 \\
\hline 0013-0029 & $\ldots$ & 2.0860 & 2200. & -27.0 & 9.37 & 0.53 & 7 \\
\hline $0019+0107$ & $\ldots$ & 2.1340 & 5700. & -28.0 & 10.48 & -0.18 & 7 \\
\hline $0058+0155$ & $\ldots$ & 1.9590 & 4200. & -28.0 & 10.19 & 0.08 & 7 \\
\hline 0150-2015 & $\ldots$ & 2.1470 & 2800. & -28.0 & 9.86 & 0.44 & 7 \\
\hline $0206+0008$ & $\ldots$ & 1.8900 & 4100. & -28.0 & 10.16 & 0.09 & 7 \\
\hline $0348+0610$ & $\ldots$ & 2.0600 & 3400. & -29.0 & 10.30 & 0.39 & 7 \\
\hline $0848+1623$ & $\ldots$ & 2.0090 & 6100. & -28.0 & 10.52 & -0.24 & 7 \\
\hline $0856+4649$ & $\ldots$ & 0.9240 & 6400. & -27.0 & 10.08 & -0.49 & 7 \\
\hline $0920+5800$ & $\ldots$ & 1.3760 & 3600. & -27.0 & 9.68 & 0.05 & 7 \\
\hline $0932+5006$ & $\ldots$ & 1.8800 & 6500. & -28.0 & 10.56 & -0.31 & 7 \\
\hline $0946+3009$ & $\ldots$ & 1.2160 & 3700. & -28.0 & 9.95 & 0.13 & 7 \\
\hline $0957+5542$ & $\ldots$ & 2.1000 & 2500 & -28.0 & 9.76 & 0.54 & 7 \\
\hline $1206+4557$ & $\ldots$ & 1.1580 & 4300 . & -28.0 & 10.07 & 0.00 & 7 \\
\hline $1222+0223$ & $\ldots$ & 2.0500 & 3100. & -28.0 & 9.94 & 0.35 & 7 \\
\hline $1228+0750$ & $\ldots$ & 1.8130 & 2400 & -28.0 & 9.68 & 0.55 & 7 \\
\hline $1245+3431$ & $\ldots$ & 2.0680 & 2800. & -27.0 & 9.57 & 0.31 & 7 \\
\hline $1246+3746$ & $\ldots$ & 2.2120 & 5500. & -27.0 & 10.18 & -0.26 & 7 \\
\hline $1248+4007$ & $\ldots$ & 1.3030 & 3400. & -27.0 & 9.62 & 0.09 & 7 \\
\hline $1254+0443$ & $\ldots$ & 1.0240 & 8300. & -27.0 & 10.33 & -0.71 & 7 \\
\hline $1317+5203$ & $\ldots$ & 1.0220 & 4700. & -27.0 & 9.84 & -0.21 & 7 \\
\hline $1321+2925$ & $\ldots$ & 1.3300 & 5000. & -26.0 & 9.68 & -0.36 & 7 \\
\hline $1338+4138$ & $\ldots$ & 1.2190 & 3900. & -28.0 & 10.00 & 0.09 & 7 \\
\hline $1407+2632$ & $\ldots$ & 0.9440 & 8200. & -27.0 & 10.30 & -0.71 & 7 \\
\hline $1416+0906$ & $\ldots$ & 2.0000 & 2800 & -28.0 & 9.84 & 0.43 & 7 \\
\hline $1421+3305$ & $\ldots$ & 1.9040 & 2700. & -28.0 & 9.80 & 0.46 & 7 \\
\hline $1522+1009$ & $\ldots$ & 1.3210 & 6000 & -28.0 & 10.39 & -0.28 & 7 \\
\hline $1634+7037$ & $\ldots$ & 1.3340 & 3400. & -29.0 & 10.18 & 0.34 & 7 \\
\hline $1704+7101$ & $\ldots$ & 2.0000 & 4500. & -28.0 & 10.26 & 0.02 & 7 \\
\hline $1715+5331$ & $\ldots$ & 1.9290 & 5000. & -29.0 & 10.62 & 0.04 & 7 \\
\hline $1718+4807$ & $\ldots$ & 1.0840 & 2600. & -28.0 & 9.62 & 0.43 & 7 \\
\hline $2302+0255$ & $\ldots$ & 1.0440 & 7100. & -27.0 & 10.20 & -0.57 & 7 \\
\hline 2340-0019 & $\ldots$ & 0.8960 & 4000. & -27.0 & 9.67 & -0.09 & 7 \\
\hline
\end{tabular}

Note. - References: 1. Forster et al. (2002); 2. Kuraszkiewicz et al. (2002); 3. Kuraszkiewicz et al. (2004); 4. Kaspi et al. (2000), after Wandel et al. (1999); 5. Kaspi et al. (2000); 6. Shang et al. (2003); 7. Brotherton et al. (1994a). All luminosities are for $H_{0}=75 \mathrm{~km} \mathrm{~s}^{-1} \mathrm{Mpc}^{-1}$ and $q_{0}=0.5$. 